\title{
THE INTERSECTION OF SUBGROUPS IN FREE GROUPS AND LINEAR PROGRAMMING
}

\author{
S. V. IVANOV
}

\begin{abstract}
We study the intersection of finitely generated subgroups of free groups by utilizing the method of linear programming. We prove that if $H_{1}$ is a finitely generated subgroup of a free group $F$, then the WN-coefficient $\sigma\left(H_{1}\right)$ of $H_{1}$ is rational and can be computed in deterministic exponential time in the size of $H_{1}$. This coefficient $\sigma\left(H_{1}\right)$ is the minimal nonnegative real number such that, for every finitely generated subgroup $\mathrm{H}_{2}$ of $F$, it is true that $\overline{\mathrm{r}}\left(H_{1}, H_{2}\right) \leq \sigma\left(H_{1}\right) \overline{\mathrm{r}}\left(H_{1}\right) \overline{\mathrm{r}}\left(H_{2}\right)$, where $\overline{\mathrm{r}}(H):=\max (\mathrm{r}(H)-1,0)$ is the reduced rank of $H, \mathrm{r}(H)$ is the rank of $H$, and $\overline{\mathrm{r}}\left(H_{1}, H_{2}\right)$ is the reduced rank of the generalized intersection of $H_{1}$ and $H_{2}$. We also show the existence of a subgroup $H_{2}^{*}=H_{2}^{*}\left(H_{1}\right)$ of $F$ such that $\overline{\mathrm{r}}\left(H_{1}, H_{2}^{*}\right)=\sigma\left(H_{1}\right) \overline{\mathrm{r}}\left(H_{1}\right) \overline{\mathrm{r}}\left(H_{2}^{*}\right)$, the Stallings graph $\Gamma\left(H_{2}^{*}\right)$ of $H_{2}^{*}$ has at most doubly exponential size in the size of $H_{1}$ and $\Gamma\left(H_{2}^{*}\right)$ can be constructed in exponential time in the size of $H_{1}$.
\end{abstract}

\section{INTRODUCTION}

Let $F$ be a finitely generated free group, let $\mathrm{r}(F)$ denote the rank of $F$ and let $\overline{\mathrm{r}}(F):=\max (\mathrm{r}(F)-1,0)$ denote the reduced rank of $F$. Let $H_{1}$ and $H_{2}$ be finitely generated subgroups of $F$. Hanna Neumann [18] proved that

$$
\overline{\mathrm{r}}\left(H_{1} \cap H_{2}\right) \leq 2 \overline{\mathrm{r}}\left(H_{1}\right) \overline{\mathrm{r}}\left(H_{2}\right)
$$

and conjectured that $\overline{\mathrm{r}}\left(H_{1} \cap H_{2}\right) \leq \overline{\mathrm{r}}\left(H_{1}\right) \overline{\mathrm{r}}\left(H_{2}\right)$.

These result and conjecture of Hanna Neumann were strengthened by Walter Neumann [19] by considering a generalized intersection of $H_{1}$ and $H_{2}$. Let $S\left(H_{1}, H_{2}\right)$ denote a set of representatives of those double cosets $H_{1} t H_{2}$ of $F, t \in F$, that have the property $H_{1} \cap t H_{2} t^{-1} \neq\{1\}$. Walter Neumann [19] proved that the set $S\left(H_{1}, H_{2}\right)$ is finite, the reduced rank $\overline{\mathrm{r}}\left(H_{1}, H_{2}\right)$ of the generalized intersection of $H_{1}$ and $H_{2}$ satisfies

$$
\overline{\mathrm{r}}\left(H_{1}, H_{2}\right):=\sum_{s \in S\left(H_{1}, H_{2}\right)} \overline{\mathrm{r}}\left(H_{1} \cap s H_{2} s^{-1}\right) \leq 2 \overline{\mathrm{r}}\left(H_{1}\right) \overline{\mathrm{r}}\left(H_{2}\right),
$$

and he conjectured that

$$
\overline{\mathrm{r}}\left(H_{1}, H_{2}\right)=\sum_{s \in S\left(H_{1}, H_{2}\right)} \overline{\mathrm{r}}\left(H_{1} \cap s H_{2} s^{-1}\right) \leq \overline{\mathrm{r}}\left(H_{1}\right) \overline{\mathrm{r}}\left(H_{2}\right) .
$$

This strengthened version of the Hanna Neumann conjecture was proved by Friedman [7] and Mineyev [17, see also Dicks's proof [3] and a proof in [13.

2010 Mathematics Subject Classification. Primary 20E05, 20E07, 20F65; Secondary 68Q25, $90 \mathrm{C} 90$.

Key words and phrases. Free groups, intersection of subgroups, rank, linear programming.

Supported in smaller part by the NSF under grant DMS 09-01782. 
Now suppose that $H_{1}$ is a fixed finitely generated subgroup of $F$. We will say that a real number $\sigma\left(H_{1}\right) \geq 0$ is the Walter Neumann coefficient for $H_{1}$, or, briefly, the WN-coefficient for $H_{1}$, if, for every finitely generated subgroup $H_{2}$ of $F$, we have

$$
\overline{\mathrm{r}}\left(H_{1}, H_{2}\right) \leq \sigma\left(H_{1}\right) \overline{\mathrm{r}}\left(H_{1}\right) \overline{\mathrm{r}}\left(H_{2}\right)
$$

and $\sigma\left(H_{1}\right)$ is minimal with this property. It is clear that if $H_{1}$ is noncyclic then

$$
\sigma\left(H_{1}\right)=\sup _{H_{2}}\left\{\frac{\overline{\mathrm{r}}\left(H_{1}, H_{2}\right)}{\overline{\mathrm{r}}\left(H_{1}\right) \overline{\mathrm{r}}\left(H_{2}\right)}\right\}
$$

over all finitely generated noncyclic subgroups $\mathrm{H}_{2}$ of $F$.

In this article, we are concerned with algorithmic computability of the WNcoefficient $\sigma\left(H_{1}\right)$ for a finitely generated subgroup $H_{1}$ of $F$ and with other properties of this number $\sigma\left(H_{1}\right)$. Utilizing the method of linear programming, we will prove the following.

Theorem 1.1. Suppose that $F$ is a free group of finite rank and $H_{1}$ is a finitely generated noncyclic subgroup of $F$. Then the following are true.

(a) There exists a linear programming problem (LP-problem) associated with $H_{1}$

$$
\mathcal{P}\left(H_{1}\right)=\max \{c x \mid A x \leq b\}
$$

with integer coefficients whose solution is equal to $-\sigma\left(H_{1}\right) \overline{\mathrm{r}}\left(H_{1}\right)$.

(b) There is a finitely generated subgroup $H_{2}^{*}$ of $F, H_{2}^{*}=H_{2}^{*}\left(H_{1}\right)$ which corresponds to a vertex solution of the dual problem

$$
\mathcal{P}^{*}\left(H_{1}\right)=\min \left\{b^{\top} y \mid A^{\top} y=c^{\top}, y \geq 0\right\}
$$

of the primal LP-problem (1.4) such that $\overline{\mathrm{r}}\left(H_{1}, H_{2}^{*}\right)=\sigma\left(H_{1}\right) \overline{\mathrm{r}}\left(H_{1}\right) \overline{\mathrm{r}}\left(H_{2}^{*}\right)$. In particular, the WN-coefficient $\sigma\left(H_{1}\right)$ of $H_{1}$ is rational and satisfies $\frac{1}{\mathrm{r}(F)} \leq \sigma\left(H_{1}\right) \leq 1$.

Furthermore, if $\Gamma\left(H_{1}\right)$ and $\Gamma\left(H_{2}^{*}\right)$ denote the Stallings graphs representing the subgroups $H_{1}$ and $H_{2}^{*}$, resp., $|E \Gamma|$ denotes the number of oriented edges in the graph $\Gamma$, and $m-1$ is the rank of $F$, then the size of $\Gamma\left(H_{2}^{*}\right)$ is at most doubly exponential in the size of $\Gamma\left(H_{1}\right)$, specifically,

$$
\left|E \Gamma\left(H_{2}^{*}\right)\right|<2^{2^{\left|E \Gamma\left(H_{1}\right)\right| / 2+2 \log _{2} m}} .
$$

(c) Assume that $H_{1}$ is given by a finite generating set or by its Stallings graph. Then, in deterministic exponential time in the size of the input, one can write down and solve the LP-problem (1.4) associated with $H_{1}$. In particular, the $W N$ coefficient $\sigma\left(H_{1}\right)$ of $H_{1}$ is computable in deterministic exponential time in the size of the input.

In addition, the Stallings graph $\Gamma\left(H_{2}^{*}\right)$ of the subgroup $H_{2}^{*}$ of part (b) can be constructed in deterministic exponential time in the size of the input.

We remark that the results similar to those of Theorem 1.1 are obtained by the author 14. for factor-free subgroups of free products of finite groups. However, the arguments of [14 do not apply to free products of infinite groups and here we develop analogous techniques suitable for free groups. For a generalization of the conjecture (1.2) to subgroups of free products of groups and relevant results, the reader is referred to articles [5, 6], 11, [13, [14.

Similarly to 14, the correspondence between subgroups $H_{2}$ and vectors $y\left(H_{2}\right)$ of the feasible polyhedron $\left\{y \mid A^{\top} y=c^{\top}, y \geq 0\right\}$ of the dual LP-problem $\mathcal{P}^{*}\left(H_{1}\right)$, 
mentioned in part (b) of Theorem 1.1. plays an important role in proofs and is reminiscent of the correspondence between (resp. almost) normal surfaces in 3dimensional manifolds and their (resp. almost) normal vectors in the Haken theory of normal surfaces and its generalizations, see [8, 9, [10, 12, 15. In particular, the idea of a vertex solution works equally well both in the context of almost normal surfaces [12, see also [9], 15], and in the context of subgroups of free groups, providing in either situation both the connectedness of the underlying object associated with a vertex solution and an upper bound on the size of the underlying object.

It is worthwhile to mention that our construction of the Stallings graph $\Gamma\left(H_{2}^{*}\right)$ in part (c) of Theorem 1.1 is somewhat succinct (cf. the definition of succinct representations of graphs, see [20]) in the sense that, despite the fact that the size of $\Gamma\left(H_{2}^{*}\right)$ could be doubly exponential, we are able to give a description of $\Gamma\left(H_{2}^{*}\right)$ in exponential time. In particular, the vertices of $\Gamma\left(H_{2}^{*}\right)$ are represented by exponentially long bit strings and the edges of $\Gamma\left(H_{2}^{*}\right)$ are drawn in blocks. As a result, we can find out in exponential time whether two given vertices of $\Gamma\left(H_{2}^{*}\right)$ are connected by an edge.

In view of Theorem 1.1, it is of interest to look at two properties of finitely generated subgroups of free groups introduced by Dicks and Ventura 4. Recall that a finitely generated subgroup $H$ of a free group $F$ is called compressed, see [4, if, for every subgroup $K$ of $F$ such that $H \subseteq K$, we have $\overline{\mathrm{r}}(H) \leq \overline{\mathrm{r}}(K)$. A finitely generated subgroup $H$ of a free group $F$ is called inert, see 4, if for every subgroup $K$ of $F$, one has $\overline{\mathrm{r}}(H \cap K) \leq \overline{\mathrm{r}}(K)$. It is immediate from the definitions that every inert subgroup is compressed. The problem whether every compressed subgroup is inert is stated by Dicks and Ventura [4] and it is still unresolved.

We say that a finitely generated subgroup $H$ of a free group $F$ is strongly inert if, for every subgroup $K$ of $F$, we have $\overline{\mathrm{r}}(H, K) \leq \overline{\mathrm{r}}(K)$. Clearly, a strongly inert subgroup is inert. It would be of interest to find an example, if it exists, to distinguish between these two classes of inert and strongly inert subgroups and, more generally, to find a finitely generated noncyclic subgroup $H$ of $F$ such that

$$
\sup _{K}\left\{\frac{\overline{\mathrm{r}}(H \cap K)}{\overline{\mathrm{r}}(H) \overline{\mathrm{r}}(K)}\right\}<\sigma(H),
$$

where the supremum, as before, is taken over all finitely generated noncyclic subgroups $K$ of $F$. Another natural question is to find an algorithm that computes this number $\sup _{K}\left\{\frac{\overline{\mathrm{r}}(H \cap K)}{\overline{\mathrm{r}}(H) \overline{\mathrm{r}}(K)}\right\}$ which could be called the Hanna Neumann coefficient of $H$.

While we are not able to distinguish between these three classes of compressed, inert, and strongly inert subgroups of $F$, our algorithms and their running times, that recognize two of these classes, are quite different.

Theorem 1.2. Suppose that $F$ is a free group of finite rank and $H$ is a finitely generated noncyclic subgroup of $F$ given by a finite generating set or by its Stallings graph. Then the following hold true.

(a) There is an algorithm that decides, in deterministic exponential time in the size of $H$, whether $H$ is strongly inert.

(b) There is an algorithm that verifies, in nondeterministic polynomial time in the size of $H$, whether $H$ is not compressed. 
Summarizing, we see that the decision problem that inquires whether a finitely generated subgroup $H$ of $F$ is strongly inert is in EXP, the decision problem that asks whether $H$ is inert is not known to be decidable, and the decision problem that inquires whether $H$ is compressed is in coNP (for the definition of computational complexity classes EXP, coNP see [1] or [20]).

\section{Preliminaries}

Suppose that $X$ is a graph. Let $V X$ denote the set of vertices of $X$ and let $E X$ be the set of oriented edges of $X$. If $e \in E X$ then $e^{-1}$ denotes the edge with the opposite orientation, $e^{-1} \neq e$.

For $e \in E X$, let $e_{-}$and $e_{+}$denote the initial and terminal, resp., vertices of $e$. A path $p=e_{1} \cdots e_{k}$, where $e_{i} \in E X,\left(e_{i}\right)_{+}=\left(e_{i+1}\right)_{-}, i=1, \ldots, k-1$, is called reduced if, for every $i=1, \ldots, k-1$, one has $e_{i} \neq e_{i+1}^{-1}$. The length of a path $p=e_{1} \cdots e_{k}$ is $k$, denoted $|p|=k$. The initial vertex of $p$ is $p_{-}=\left(e_{1}\right)_{-}$and the terminal vertex of $p$ is $p_{+}=\left(e_{k}\right)_{+}$. A path $p$ is closed if $p_{-}=p_{+}$.

If $p=e_{1} \cdots e_{k}$ is a closed path, then a cyclic permutation $\bar{p}$ of $p$ is a path of the form $e_{1+i} e_{2+i} \cdots e_{k+i}$, where $i=0,1, \ldots, k$ and the indices are considered $\bmod k$. The subgraph of $X$ that consists of edges of all closed paths $p$ of $X$ such that $|p|>0$ and every cyclic permutation of $p$ is reduced, is called the core of $X$, denoted $\operatorname{core}(X)$.

Let $U$ be a finite connected graph such that $\operatorname{core}(U)=U$, let $o \in V U$ and let $F=\pi_{1}(U, o)$ be the fundamental group of $U$ at $o$. Then $F$ is a free group of rank $\mathrm{r}(F)=|E U| / 2-|V U|+1$, where $|B|$ is the cardinality of a finite set $B$, and the elements of $F$ can be thought of as reduced closed paths in $U$ starting at $o$.

Following Stallings [22, see also [2], 16], with every (finitely generated) subgroup $H$ of $F_{U}=\pi_{1}(U, o)$, we can associate a (resp. finite) graph $Y=Y(H)$ and a map $\beta: Y \rightarrow U$ of graphs so that $H$ is isomorphic to $\pi_{1}\left(Y, o_{Y}\right)$, where $o_{Y} \in V Y$, $\beta\left(o_{Y}\right)=o$, and a reduced path $p \in \pi_{1}(U, o)$ belongs to $H$ if and only if there is a reduced path $p_{H} \in \pi_{1}\left(Y, o_{Y}\right)$ such that $\beta\left(p_{H}\right)=p$. In addition, we may assume that $\beta$ is a locally injective map of graphs, i.e., the restriction of $\beta$ on a regular neighborhood of every vertex of $Y$ is injective. We call a locally injective map of graphs an immersion. Since $\beta$ is an immersion, it follows that every reduced path in $H \subseteq \pi_{1}(U, o)$ has a unique preimage in $Y$.

Consider two finitely generated subgroups $H_{1}$ and $H_{2}$ of the free group $F_{U}=$ $\pi_{1}(U, o)$. Pick a set $S\left(H_{1}, H_{2}\right)$ of representatives of those double cosets $H_{1} g H_{2}$, $g \in F_{U}$, for which the intersection $H_{1} \cap g H_{2} g^{-1}$ is nontrivial.

Let $Y_{1}, Y_{2}$ be Stallings graphs of the subgroups $H_{1}, H_{2}$ and let $Y_{1} \underset{U}{\times} Y_{2}$ denote the pullback of the maps $\beta_{i}: Y_{i} \rightarrow U, i=1,2$. Recall that

$$
\begin{aligned}
& V\left(Y_{1} \underset{U}{\times} Y_{2}\right)=\left\{\left(v_{1}, v_{2}\right) \mid v_{i} \in V Y_{i}, \beta_{1}\left(v_{1}\right)=\beta_{2}\left(v_{2}\right)\right\}, \\
& E\left(Y_{1} \underset{U}{\times} Y_{2}\right)=\left\{\left(e_{1}, e_{2}\right) \mid e_{i} \in E Y_{i}, \beta_{1}\left(e_{1}\right)=\beta_{2}\left(e_{2}\right)\right\},
\end{aligned}
$$

and $\left(e_{1}, e_{2}\right)_{-}=\left(\left(e_{1}\right)_{-},\left(e_{2}\right)_{-}\right),\left(e_{1}, e_{2}\right)_{+}=\left(\left(e_{1}\right)_{+},\left(e_{2}\right)_{+}\right)$.

According to Walter Neumann [19, the set $S\left(H_{1}, H_{2}\right)$ is finite and the nontrivial intersections $H_{1} \cap s H_{2} s^{-1}$, where $s \in S\left(H_{1}, H_{2}\right)$, are in bijective correspondence 
with the connected components $W_{s}$ of the core $W:=\operatorname{core}\left(Y_{1} \underset{U}{\times} Y_{2}\right)$. Moreover, for every $s \in S\left(H_{1}, H_{2}\right)$, we have

$$
\overline{\mathrm{r}}\left(H_{1} \cap s H_{2} s^{-1}\right)=\overline{\mathrm{r}}\left(W_{s}\right)=\left|E W_{s}\right| / 2-\left|V W_{s}\right| .
$$

Hence,

$$
\sum_{s \in S\left(H_{1}, H_{2}\right)} \overline{\mathrm{r}}\left(H_{1} \cap s H_{2} s^{-1}\right)=\overline{\mathrm{r}}(W)=|E W| / 2-|V W| .
$$

Let $\alpha_{i}^{\prime}$ denote the projection map $\underset{1}{Y_{1}} \times Y_{2} \rightarrow Y_{i}, i=1,2$, i.e., $\alpha_{i}^{\prime}\left(\left(e_{1}, e_{2}\right)\right)=e_{i}$ and $\alpha_{i}^{\prime}\left(\left(v_{1}, v_{2}\right)\right)=v_{i}$. Restricting $\alpha_{i}^{\prime}$ on $W \subseteq Y_{1} \underset{U}{\times} Y_{2}$, we obtain the map $\alpha_{i}: W \rightarrow Y_{i}$, $i=1,2$. In this notation, we have a commutative diagram depicted in Figure 1.

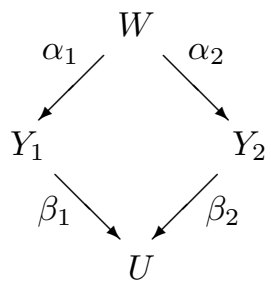

Figure 1

In particular, if $X \in\left\{Y_{1}, Y_{2}, W, U\right\}$, then there is a canonical immersion

$$
\varphi: X \rightarrow U
$$

where $\varphi=\beta_{i}$ if $X=Y_{i}, i=1,2, \varphi=\operatorname{id}_{U}$ if $X=U$ and $\varphi=\beta_{i} \alpha_{i}$ if $X=W$. More generally, we will say that $X$ is a $U$-graph if $X$ is equipped with a graph map $\varphi: X \rightarrow U$. A $U$-graph $X$ is reduced if $\varphi$ is an immersion. For example, $Y_{1}, Y_{2}, W, U$ are reduced $U$-graphs. If $x \in V X \cup E X$ then $\varphi(x) \in V U \cup E U$ is called the label of $x$.

It will be convenient to work with a graph $U$ of a special form which we denote $U_{m}$. The graph $U_{m}$ contains two vertices $o_{1}, o_{2}, V U_{m}:=\left\{o_{1}, o_{2}\right\}$, and the vertices $o_{1}, o_{2}$ are connected by $m \geq 3$ nonoriented edges so that the oriented edges $a_{1}, \ldots, a_{m} \in E U_{m}$ start at $o_{1}$ and end in $o_{2}$, see Figure 2, where the case $m=3$ is depicted.

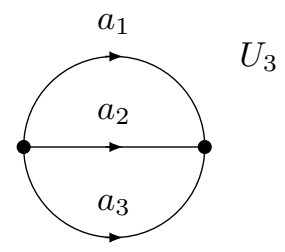

Figure 2

We will be considering mostly $U_{m}$-graphs, where $m \geq 3$. Since $U_{m}$ is fixed, we will be writing $Y_{1} \times Y_{2}$ in place of $Y_{1} \underset{U_{m}}{\times} Y_{2}$.

Denote

$$
\mathcal{A}:=\left\{a_{1}, \ldots, a_{m}\right\}
$$


Let $X$ be a $U_{m}$-graph. A vertex $v \in V X$ is called an $i$-vertex if $\varphi(v)=o_{i}$, $i=1,2$. Clearly, if $e_{+}$is a 2 -vertex then $\varphi(e) \in \mathcal{A}$ and if $e_{+}$is a 1-vertex then $\varphi(e) \in \mathcal{A}^{-1}=\left\{a_{1}^{-1}, \ldots, a_{m}^{-1}\right\}$.

An edge $e \in E X$ is called a $b$-edge, where $b \in \mathcal{A}$, if $\varphi(e)=b$. The set of all $b$-edges of $X$ is denoted $E_{b} X$. Clearly,

$$
\sum_{b \in \mathcal{A}}\left|E_{b} X\right|=|E X| / 2
$$

\section{The System of Linear Inequalities SLI $\left[Y_{1}\right]$}

Suppose that $Y_{1}$ is a finite reduced $U_{m}$-graph such that $Y_{1}=\operatorname{core}\left(Y_{1}\right)$ and

$$
\overline{\mathrm{r}}\left(Y_{1}\right):=-\chi\left(Y_{1}\right)=\left|E Y_{1}\right| / 2-\left|V Y_{1}\right|>0,
$$

where $\chi\left(Y_{1}\right)$ is the Euler characteristic of $Y_{1}$ (since $E Y_{1}$ is the set of oriented edges of $Y_{1}$, we use $\left|E Y_{1}\right| / 2$ in $\left.\chi\left(Y_{1}\right)\right)$. This graph $Y_{1}$ will be held fixed throughout Sections $3-4$.

Let $\left(A_{1}, \ldots, A_{m}\right)$ be an $m$-tuple of sets $A_{j}$ such that

$$
A_{j} \subseteq E_{a_{j}} Y_{1}, \quad j=1, \ldots, m .
$$

Recall that $m=|\mathcal{A}|$ and $m \geq 3$, see (2.1).

Let $e, f \in \bigcup_{j=1}^{m} A_{j}$. We say that the edges $e, f$ are $i$-related, written $e \sim_{i} f$, if $e_{-}=f_{-}$in $Y_{1}$ when $i=1$ or $e_{+}=f_{+}$in $Y_{1}$ when $i=2$. Note that it follows from (3.1) and from $Y_{1}$ being a $U_{m}$-graph that $e_{-}, f_{-}$are 1-vertices while $e_{+}, f_{+}$ are 2 -vertices. Clearly, $\sim_{i}$ is an equivalence relation on the set $\bigcup_{j=1}^{m} A_{j}$.

Let $[e]_{\sim_{i}}$ denote the equivalence class of an edge $e \in \bigcup_{j=1}^{m} A_{j}$ relative to this equivalence relation $\sim_{i}$ and let $\left|[e]_{\sim_{i}}\right|$ denote the cardinality of $[e]_{\sim_{i}}$.

We will say that an $m$-tuple $\left(A_{1}, \ldots, A_{m}\right)$ is $i$-admissible, where $i=1,2$ is fixed, if the union $\bigcup_{j=1}^{m} A_{j}$ is not empty and, for every $e \in \bigcup_{j=1}^{m} A_{j}$, we have $\left|[e]_{\sim_{i}}\right|>1$. It is clear that, for every $e \in \bigcup_{j=1}^{m} A_{j}$,

$$
2 \leq\left|[e]_{\sim_{i}}\right| \leq k \leq m,
$$

where $k$ is the number of nonempty sets $A_{j}$ in the tuple $\left(A_{1}, \ldots, A_{m}\right)$.

If $\left(A_{1}, \ldots, A_{m}\right)$ is an $i$-admissible tuple, we define the number $N_{i}\left(A_{1}, \ldots, A_{m}\right)$ to be the sum

$$
\sum\left(\left|[e]_{\sim_{i}}\right|-2\right)
$$

over all equivalence classes $[e]_{\sim_{i}}$ of the equivalence relation $\sim_{i}$ on $\bigcup_{j=1}^{m} A_{j}$. Hence,

$$
N_{i}\left(A_{1}, \ldots, A_{m}\right):=\sum_{[e]_{\sim_{i}}}\left(\left|[e]_{\sim_{i}}\right|-2\right)
$$

We note that

$$
\overline{\mathrm{r}}\left(Y_{1}\right)=\left|E Y_{1}\right| / 2-\left|V Y_{1}\right|=\frac{1}{2} \sum_{u \in V Y_{1}}(\operatorname{deg} u-2),
$$

where $\operatorname{deg} u$ is the degree of a vertex $u \in V Y_{1}$, i.e., $\operatorname{deg} u$ is the number of edges $e \in E Y_{1}$ such that $e_{+}=v$.

Let $V_{i} Y_{1}$ denote the set of all $i$-vertices of $Y_{1}, i=1,2$. Define

$$
\overline{\mathrm{r}}_{i}\left(Y_{1}\right):=\frac{1}{2} \sum_{u \in V_{i} Y_{1}}(\operatorname{deg} u-2) \text {. }
$$


Observe that $\overline{\mathrm{r}}\left(Y_{1}\right)=\overline{\mathrm{r}}_{1}\left(Y_{1}\right)+\overline{\mathrm{r}}_{2}\left(Y_{1}\right)$ and that

$$
\begin{aligned}
N_{i}\left(A_{1}, \ldots, A_{m}\right) & :=\sum_{[e]_{\sim_{i}}}\left(\left|[e]_{\sim_{i}}\right|-2\right) \\
& \leq \sum_{u \in V_{i} Y_{1}}(\operatorname{deg} u-2)=2 \overline{\mathrm{r}}_{i}\left(Y_{1}\right) \leq 2 \overline{\mathrm{r}}\left(Y_{1}\right) .
\end{aligned}
$$

For every nonempty set $B \subseteq E_{a_{j}} Y_{1}$, we consider a variable $x_{j, B}$. We also introduce a special variable $x_{s}$. Note that, for given $j$, the set of all variables $x_{j, B}$ is finite and its cardinality is equal to $2^{\left|E_{a_{j}} Y_{1}\right|}-1$.

Now we will define a system of inequalities in these variables $x_{j, B}, x_{s}$ so that each inequality is determined by means of an $i$-admissible tuple $\left(A_{1}, \ldots, A_{m}\right)$.

For an $i$-admissible tuple $\left(A_{1}, \ldots, A_{m}\right)$, let $A_{j_{1}}, \ldots, A_{j_{k}}$ denote all nonempty sets in $\left(A_{1}, \ldots, A_{m}\right)$.

If $i=1$ then the inequality, corresponding to the 1 -admissible tuple $\left(A_{1}, \ldots, A_{m}\right)$, is defined as follows

$$
-x_{j_{1}, A_{j_{1}}}-x_{j_{2}, A_{j_{2}}}-\ldots-x_{j_{k}, A_{j_{k}}}-(k-2) x_{s} \leq-N_{1}\left(A_{1}, \ldots, A_{m}\right) .
$$

If $i=2$ then the inequality, corresponding to the 2 -admissible tuple $\left(A_{1}, \ldots, A_{m}\right)$, is defined as follows

$$
x_{j_{1}, A_{j_{1}}}+x_{j_{2}, A_{j_{2}}}+\ldots+x_{j_{k}, A_{j_{k}}}-(k-2) x_{s} \leq-N_{2}\left(A_{1}, \ldots, A_{m}\right) .
$$

Let

$$
\operatorname{SLI}\left[Y_{1}\right]
$$

denote the system of all linear inequalities (3.5)-(3.6) constructed for all $i$-admissible tuples $\left(A_{1}, \ldots, A_{m}\right), i=1,2$. Clearly, $\operatorname{SLI}\left[Y_{1}\right]$ is finite.

Assume that the map

$$
\alpha_{2}: \operatorname{core}\left(Y_{1} \times Y_{2}\right) \rightarrow Y_{2}
$$

is surjective, i.e., $\alpha_{2}\left(\operatorname{core}\left(Y_{1} \times Y_{2}\right)\right)=Y_{2}$.

For every $i$-vertex $u \in V Y_{2}$, consider all the edges $e_{1}, \ldots, e_{k} \in E Y_{2}$ such that $\left(e_{1}\right)_{-}=\cdots=\left(e_{k}\right)_{-}=u$ if $i=1$ or $\left(e_{1}\right)_{+}=\cdots=\left(e_{k}\right)_{+}=u$ if $i=2$, so $\varphi\left(e_{1}\right), \ldots, \varphi\left(e_{k}\right) \in \mathcal{A}$, see (2.1), and the edges $e_{1}, \ldots, e_{k}$ start or finish at $u$. Denote $\varphi\left(e_{\ell}\right)=a_{j_{\ell}}$ for $\ell=1, \ldots, k$.

If $j \notin\left\{j_{1}, \ldots, j_{k}\right\}$, we set $A_{j}(u):=\varnothing$. Otherwise, $j=j_{\ell}$ for some $\ell=1, \ldots, k$, and we set

$$
A_{j_{\ell}}(u):=\alpha_{1} \alpha_{2}^{-1}\left(e_{\ell}\right) \subseteq E_{j_{\ell}} Y_{1},
$$

where $\alpha_{2}^{-1}\left(e_{\ell}\right)$ is the full preimage of the edge $e_{\ell}$ in core $\left(Y_{1} \times Y_{2}\right)$. It is immediate from the definitions that the tuple $\left(A_{1}(u), \ldots, A_{m}(u)\right)$ is $i$-admissible. Recall that the graphs $Y_{1}$ and $Y_{2}$ coincide with their cores and have no vertices of degree less than 2 .

Since every $i$-admissible tuple $\left(A_{1}, \ldots, A_{m}\right)$ gives rise to an inequality (3.5) if $i=1$ or to an inequality (3.6) if $i=2$ and every $i$-vertex $u \in V Y_{2}$ defines, as indicated above, an $i$-admissible tuple $\left(A_{1}(u), \ldots, A_{m}(u)\right)$, it follows that every vertex $u \in V Y_{2}$ is mapped to a certain inequality of the system $\operatorname{SLI}\left[Y_{1}\right]$, denoted $\operatorname{inq}_{V}(u)$. Thus we obtain a function

$$
\operatorname{inq}_{V}: V Y_{2} \rightarrow \operatorname{SLI}\left[Y_{1}\right]
$$


from the set $V Y_{2}$ of vertices of a graph $Y_{2}$, with the property that the map

$$
\alpha_{2}: \operatorname{core}\left(Y_{1} \times Y_{2}\right) \rightarrow Y_{2}
$$

is surjective, to the set of inequalities of the system $\operatorname{SLI}\left[Y_{1}\right]$.

If $q$ is an inequality of the system $\operatorname{SLI}\left[Y_{1}\right]$, written $q \in \operatorname{SLI}\left[Y_{1}\right]$, we let $q^{L}$ denote the left hand side of $q$, let $q^{R}$ denote the number of the right hand side of the inequality $q$, and let $k(q) \geq 2$ denote the parameter $k$ for $q$, see the definition of inequalities (3.5) $-(3.6)$.

Lemma 3.1. Suppose $Y_{2}$ is a finite reduced $U_{m}$-graph with the property that the map $\alpha_{2}: \operatorname{core}\left(Y_{1} \times Y_{2}\right) \rightarrow Y_{2}$ is surjective. Then

$$
\sum_{u \in V Y_{2}} \operatorname{inq}_{V}(u)^{L}=-2 \overline{\mathrm{r}}\left(Y_{2}\right) x_{s} \quad \text { and } \sum_{u \in V Y_{2}} \operatorname{inq}_{V}(u)^{R}=-2 \overline{\mathrm{r}}\left(\operatorname{core}\left(Y_{1} \times Y_{2}\right)\right) .
$$

Proof. Suppose $e \in E Y_{2}, \varphi(e)=a_{j}$, and $e_{-}=u_{1}, e_{+}=u_{2}$. Clearly, $u_{i}$ is an $i$-vertex of $Y_{2}, i=1,2$. Denote $B:=\alpha_{1} \alpha_{2}^{-1}(e) \subseteq E_{a_{j}} Y_{1}$. Then the variables $-x_{j, B}$ and $x_{j, B}$ of $\operatorname{inq}_{V}\left(u_{1}\right)^{L}$ and $\operatorname{inq}_{V}\left(u_{2}\right)^{L}$, resp., will cancel out in the sum $\sum_{u \in V Y_{2}} \operatorname{inq}_{V}(u)^{L}$. It is easy to see that all occurrences of the variable $\pm x_{j, B}$, where $j=1, \ldots, m$, $B \subseteq E_{a_{j}} Y_{1},|B|>0$, in the sum $\sum_{u \in V Y_{2}} \operatorname{inq}_{V}(u)^{L}$ can be paired down by using edges of $Y_{2}$ as indicated above.

Now we observe that every vertex $u \in V Y_{2}$ of degree $d \geq 2$ contributes $-(d-2)$ to the coefficient of $x_{s}$ in the sum $\sum_{u \in V Y_{2}} \operatorname{inq}_{V}(u)^{L}$ and that

$$
-\chi\left(Y_{2}\right)=\overline{\mathrm{r}}\left(Y_{2}\right)=\frac{1}{2} \sum_{u \in V Y_{2}}(\operatorname{deg} u-2) .
$$

Therefore, we may conclude that

$$
\sum_{u \in V Y_{2}} \operatorname{inq}_{V}(u)^{L}=-2 \overline{\mathrm{r}}\left(Y_{2}\right) x_{s},
$$

as required.

The second equality of the Lemma's statement follows from the analogous equality

$$
-\chi(W)=\overline{\mathrm{r}}(W)=\frac{1}{2} \sum_{u \in V W}(\operatorname{deg} u-2)
$$

for $W=\operatorname{core}\left(Y_{1} \times Y_{2}\right)$ and from the definition (3.3) of the numbers $N_{i}\left(A_{1}, \ldots, A_{m}\right)$ that are used in the right hand sides of inequalities (3.5)-(3.6). Indeed, for every $v \in V Y_{2}$, the term $\operatorname{inq}_{V}(v)^{R}$ of the sum

$$
\sum_{u \in V Y_{2}} \operatorname{inq}_{V}(u)^{R}
$$

is $-N\left(A_{1}(v), \ldots, A_{m}(v)\right)$, here the sets $A_{j}(v)$ are defined as in (3.8), which in turn is equal to

$$
-\sum_{\alpha_{2}(w)=v}(\operatorname{deg} w-2)
$$

where the summation runs over all vertices $w \in W=\operatorname{core}\left(Y_{1} \times Y_{2}\right)$ with $\alpha_{2}(w)=v$. Since the map $\alpha_{2}$ is surjective, we obtain

$$
\sum_{u \in V Y_{2}} \operatorname{inq}_{V}(u)^{R}=-2 \overline{\mathrm{r}}\left(\operatorname{core}\left(Y_{1} \times Y_{2}\right)\right),
$$


as required.

Let $A$ be a finite set. A combination with repetitions $B$ of $A$, denoted

$$
B=\left[\left[b_{1}, \ldots, b_{\ell}\right]\right] \sqsubseteq A,
$$

is a finite unordered collection of multiple copies of elements of $A$. Hence, $b_{i} \in A$ and $b_{i}=b_{j}$ is possible when $i \neq j$. If $B=\left[\left[b_{1}, \ldots, b_{\ell}\right]\right]$ is a combination with repetitions then the cardinality $|B|$ of $B$ is $|B|:=\ell$.

Observe that the graph $Y_{2}$ of Lemma 3.1 can be used to construct a combination with repetitions, denoted

$$
\operatorname{inq}\left(V Y_{2}\right) \text {, }
$$

of the system $\operatorname{SLI}\left[Y_{1}\right]$, whose elements are individual inequalities, so that every inequality $q=\operatorname{inq}_{V}(u)$ of $\operatorname{SLI}\left[Y_{1}\right]$ occurs in inq $\left(V Y_{2}\right)$ a number of times equal to the number of preimages of $q$ in $V Y_{2}$ under the map inq ${ }_{V}$. It follows from Lemma3.1 that if

$$
\operatorname{inq}\left(V Y_{2}\right)=\left[\left[q_{1}, \ldots, q_{\ell}\right]\right] \sqsubseteq \mathrm{SLI}\left[Y_{1}\right]
$$

then

$$
\sum_{q \in \operatorname{inq}\left(V Y_{2}\right)} q^{L}:=\sum_{i=1}^{\ell} q_{i}^{L}=-C x_{s},
$$

where $C \geq 0$ is an integer, $C=2 \overline{\mathrm{r}}\left(Y_{2}\right)$.

For convenience of references, we introduce the following property of a graph $Y_{2}$ (which need not be connected).

(B) $Y_{2}$ is a finite reduced $U_{m}$-graph such that the map $\alpha_{2}$ : $\operatorname{core}\left(Y_{1} \times Y_{2}\right) \rightarrow Y_{2}$ is surjective, $\operatorname{core}\left(Y_{2}\right)=Y_{2}$, and $\overline{\mathrm{r}}\left(Y_{2}\right)=-\chi\left(Y_{2}\right)>0$.

Note that the equality core $\left(Y_{2}\right)=Y_{2}$ could be dropped as it follows from the surjectivity of the map $\alpha_{2}: \operatorname{core}\left(Y_{1} \times Y_{2}\right) \rightarrow Y_{2}$.

Lemma 3.2. Suppose $Q$ is a nonempty combination with repetitions of $\operatorname{SLI}\left[Y_{1}\right]$ and

$$
\sum_{q \in Q} q^{L}=-C(Q) x_{s}
$$

where $C(Q)>0$ is an integer. Then there exists a finite reduced $U_{m}$-graph $Y_{2, Q}$ with property $(B)$ such that, letting $\widetilde{Q}:=\operatorname{inq}\left(V Y_{2, Q}\right)$, one has $|\widetilde{Q}|=|Q|$ and

$$
\begin{aligned}
& \sum_{q \in Q} q^{L}=\sum_{q \in \widetilde{Q}} q^{L}=-2 \overline{\mathrm{r}}\left(Y_{2, Q}\right) x_{s}, \\
& \sum_{q \in Q} q^{R} \geq \sum_{q \in \widetilde{Q}} q^{R}=-2 \overline{\mathrm{r}}\left(\operatorname{core}\left(Y_{1} \times Y_{2, Q}\right)\right) .
\end{aligned}
$$

Proof. We will construct a graph $Y_{2, Q}$ whose vertices $u_{1}, \ldots, u_{|Q|}$ are in bijective correspondence

$$
u_{i} \mapsto q_{i}, \quad i=1, \ldots,|Q|,
$$

with elements of the combination $Q=\left[\left[q_{1}, \ldots, q_{|Q|}\right]\right] \sqsubseteq \mathrm{SLI}\left[Y_{1}\right]$. 
Recall that every inequality $q_{i}$ in $Q$ has one of the form (3.5)-(3.6). It follows from the assumption (3.9) that all terms $\pm x_{j, D}$ in the sum

$$
\sum_{i=1}^{|Q|} q_{i}^{L}
$$

will cancel out. Hence, there exists an involution $\iota$ on the set of all terms $\pm x_{j, D}$ of the formal sum (3.12) such that $\iota$ takes every term $\pm x_{j, D}$ of $q_{i_{1}}^{L}$ to a term $\mp x_{j, D}$ of $q_{i_{2}}^{L}$, where $i_{2} \neq i_{1}$, and $\iota^{2}=\mathrm{id}$.

If $\iota$ takes the term $-x_{j, D}$ of $q_{i_{1}}^{L}$ to the term $x_{j, D}$ of $q_{i_{2}}^{L}$, then we connect the vertex $u_{i_{1}}$ to $u_{i_{2}}$ by an oriented edge in $Y_{2, Q}$ whose label is $a_{j}, a_{j} \in \mathcal{A}$. This definition determines the local structure of the graph $Y_{2, Q}$ and, in particular, means that if $q_{i} \in Q$ has type (3.5), then $u_{i}$ is a 1 -vertex. On the other hand, if $q_{i} \in Q$ has type (3.6), then $u_{i}$ is a 2-vertex. Furthermore, it follows from this definition that the degree of every vertex $u_{i}$ of $Y_{2, Q}$ is $k\left(q_{i}\right) \geq 2$. Recall that $k\left(q_{i}\right)$ denotes the parameter $k$ for $q_{i}$, see the definitions (3.5)-(3.6).

Looking at the coefficients of $-x_{s}$ in (3.12), we can see from (3.9) and (3.5)-(3.6) that

$$
C(Q)=\sum_{i=1}^{|Q|}\left(k\left(q_{i}\right)-2\right)>0 .
$$

Hence, the graph $Y_{2, Q}$ has a vertex of degree at least 3 .

Therefore, $Y_{2, Q}$ is a finite reduced $U_{m}$-graph such that $\operatorname{core}\left(Y_{2, Q}\right)=Y_{2, Q}$ and $\overline{\mathrm{r}}\left(Y_{2, Q}\right)>0$. Note that $Y_{2, Q}$ is not uniquely determined by $Q$ (as there are many choices to define the involution $\iota$, i.e., to do cancellations in the left hand side of (3.9) ).

Consider the graph core $\left(Y_{1} \times Y_{2, Q}\right)$ and the associated graph maps

$$
\alpha_{1}: \operatorname{core}\left(Y_{1} \times Y_{2, Q}\right) \rightarrow Y_{1}, \quad \alpha_{2}: \operatorname{core}\left(Y_{1} \times Y_{2, Q}\right) \rightarrow Y_{2, Q} .
$$

It is clear from the definitions that $\alpha_{2}$ is surjective, hence, $Y_{2, Q}$ has property (B).

Let $u_{i}$ be a vertex of $Y_{2, Q}$ and let the inequality $q_{i} \in Q$, corresponding to $u_{i}$, is constructed by means of an $\alpha_{i}$-admissible tuple $\left(A_{1, i}, \ldots, A_{m, i}\right)$ so that

$$
\begin{aligned}
q_{i}^{L} & =(-1)^{\alpha_{i}} x_{j_{1, i}, A_{j_{1, i}, i}}+\cdots+(-1)^{\alpha_{i}} x_{j_{\left(q_{i}\right), i}, A_{j_{k\left(q_{i}\right), i}}}-\left(k\left(q_{i}\right)-2\right) x_{s}, \\
q_{i}^{R} & =-N_{\alpha_{i}}\left(A_{1, i}, \ldots, A_{m, i}\right) .
\end{aligned}
$$

It follows from the definitions that, for every possible term $\pm x_{j, A_{j, i}}$ of $q_{i}^{L}$, where $A_{j, i} \neq \varnothing$, the set $A_{j}\left(u_{i}\right)$, as defined in (3.8), contains $A_{j, i}$ as a subset and, if $q_{i}^{L}$ has no term $\pm x_{j, A_{j, i}}$, i.e., $A_{j, i}=\varnothing$, then $A_{j}\left(u_{i}\right)=\varnothing$. These remarks mean that if $\widetilde{Q}:=\operatorname{inq}\left(V Y_{2, Q}\right)$ then $|\widetilde{Q}|=|Q|$ and $Y_{2, Q}=Y_{2, \widetilde{Q}}$ for a suitable involution $\widetilde{\iota}=\widetilde{\iota}(\widetilde{Q})$.

Hence, if $q_{i}$ has the form (3.5), i.e., $\alpha_{i}=1$, then

$$
\operatorname{inq}_{V}\left(u_{i}\right)^{L}=-x_{j_{1, i}, A_{j_{1}}\left(u_{i}\right)}-\ldots-x_{j_{k\left(q_{i}\right), i}, A_{j_{k\left(q_{i}\right)}}\left(u_{i}\right)}-\left(k\left(q_{i}\right)-2\right) x_{s},
$$

where $A_{j_{t}, i} \subseteq A_{j_{t}}\left(u_{i}\right)$ for every $t=1, \ldots, k\left(q_{i}\right)$ and

$$
N_{1}\left(A_{1, i}, \ldots, A_{m, i}\right) \leq N_{1}\left(A_{1}\left(u_{i}\right), \ldots, A_{m}\left(u_{i}\right)\right) .
$$


Analogously, if $q_{i}$ has the form (3.6), i.e., $\alpha_{i}=2$, then

$$
\operatorname{inq}_{V}\left(u_{i}\right)^{L}=x_{j_{1, i}, A_{j_{1}}\left(u_{i}\right)}+\ldots+x_{j_{k\left(q_{i}\right), i}, A_{j_{k\left(q_{i}\right)}}\left(u_{i}\right)}-\left(k\left(q_{i}\right)-2\right) x_{s},
$$

where $A_{j_{t}, i} \subseteq A_{j_{t}}\left(u_{i}\right)$ for every $t=1, \ldots, k\left(q_{i}\right)$ and

$$
N_{2}\left(A_{1, i}, \ldots, A_{m, i}\right) \leq N_{2}\left(A_{1}\left(u_{i}\right), \ldots, A_{m}\left(u_{i}\right)\right) .
$$

Therefore,

$$
\sum_{q \in \operatorname{inq}\left(V Y_{2, Q}\right)} q^{R}=\sum_{q \in \widetilde{Q}} q^{R} \leq \sum_{q \in Q} q^{R}
$$

Now both the equality (3.10) and inequality (3.11) follow from Lemma 3.1.

We summarize Lemmas $3.1+3.2$ as follows.

Lemma 3.3. The function

$$
\text { inq : } Y_{2} \mapsto \operatorname{inq}\left(V Y_{2}\right)=Q
$$

from the set of finite reduced $U_{m}$-graphs $Y_{2}$ with property $(B)$ to the set of combinations with repetitions $Q$ of $\mathrm{SLI}\left[Y_{1}\right]$ with the property $\sum_{q \in Q} q^{L}=-C(Q) x_{s}$, where $C(Q)>0$ is an integer, is such that

$$
\sum_{q \in \operatorname{inq}\left(V Y_{2}\right)} q^{L}=-2 \overline{\mathrm{r}}\left(Y_{2}\right) x_{s} \quad \text { and } \sum_{q \in \operatorname{inq}\left(V Y_{2}\right)} q^{R}=-2 \overline{\mathrm{r}}\left(\operatorname{core}\left(Y_{1} \times Y_{2}\right)\right) .
$$

In addition, for every $Q$ in the codomain of the function inq, there exists a graph $Y_{2, Q}$ in the domain of inq such that, letting $\widetilde{Q}:=\operatorname{inq}\left(V Y_{2, Q}\right)$, one has $|\widetilde{Q}|=|Q|$ and

$$
\begin{aligned}
& \sum_{q \in Q} q^{L}=\sum_{q \in \widetilde{Q}} q^{L}=-2 \overline{\mathrm{r}}\left(Y_{2, Q}\right) x_{s} \\
& \sum_{q \in Q} q^{R} \geq \sum_{q \in \widetilde{Q}} q^{R}=-2 \overline{\mathrm{r}}\left(\operatorname{core}\left(Y_{1} \times Y_{2, Q}\right)\right) .
\end{aligned}
$$

Proof. This is straightforward from Lemmas 3.13 .2

\section{Utilizing THE METHOD OF LINEAR PROGRAMMinG}

Let us briefly review relevant results from the theory of linear programming (LP) over the field $\mathbb{Q}$ of rational numbers. Following the notation of Schrijver's monograph [21, let $A \in \mathbb{Q}^{m^{\prime} \times n^{\prime}}$ be an $m^{\prime} \times n^{\prime}$-matrix, let $b \in \mathbb{Q}^{m^{\prime} \times 1}=\mathbb{Q}^{m^{\prime}}$ be a column vector, let $c \in \mathbb{Q}^{1 \times n^{\prime}}$ be a row vector, $c=\left(c_{1}, \ldots, c_{n^{\prime}}\right)$, and let $x$ be a column vector consisting of variables $x_{1}, \ldots, x_{n^{\prime}}$, so $x=\left(x_{1}, \ldots, x_{n^{\prime}}\right)^{\top}$, where $M^{\top}$ means the transpose of a matrix $M$. The inequality $x \geq 0$ means that $x_{i} \geq 0$ for every $i$.

A typical LP-problem asks about the maximal value of the objective linear function

$$
c x=c_{1} x_{1} \cdots+c_{n^{\prime}} x_{n^{\prime}}
$$

over all $x \in \mathbb{Q}^{n^{\prime}}$ subject to the system of linear inequalities $A x \leq b$. This value (and often the LP-problem itself) is denoted

$$
\max \{c x \mid A x \leq b\} \text {. }
$$


We write $\max \{c x \mid A x \leq b\}=-\infty$ if the set $\{c x \mid A x \leq b\}$ is empty. We write $\max \{c x \mid A x \leq b\}=+\infty$ if the set $\{c x \mid A x \leq b\}$ is unbounded from above and say that $\max \{c x \mid A x \leq b\}$ is finite if the set $\{c x \mid A x \leq b\}$ is nonempty and bounded from above. The notation and terminology for an LP-problem

$$
\min \{c x \mid A x \leq b\}=-\max \{-c x \mid A x \leq b\}
$$

is analogous with $-\infty$ and $+\infty$ interchanged.

If $\max \{c x \mid A x \leq b\}$ is an LP-problem defined as above, then the problem

$$
\min \left\{b^{\top} y \mid A^{\top} y=c^{\top}, y \geq 0\right\},
$$

where $y=\left(y_{1}, \ldots, y_{m^{\prime}}\right)^{\top}$, is called the dual problem of $\max \{c x \mid A x \leq b\}$.

The (weak) duality theorem of linear programming can now be stated as follows, see [21, Sections 7.4, 14.3].

Theorem A. Let $\max \{c x \mid A x \leq b\}$ be an LP-problem and let

$$
\min \left\{b^{\top} y \mid A^{\top} y=c^{\top}, y \geq 0\right\}
$$

be its dual LP-problem. Then for every $x \in \mathbb{Q}^{n^{\prime}}$ such that $A x \leq b$ and every $y \in \mathbb{Q}^{m^{\prime}}$ such that $A^{\top} y=c^{\top}, y \geq 0$, one has that $c x=y^{\top} A x \leq b^{\top} y$ and

$$
\max \{c x \mid A x \leq b\}=\min \left\{b^{\top} y \mid A^{\top} y=c^{\top}, y \geq 0\right\}
$$

provided both polyhedra $\{x \mid A x \leq b\}$ and $\left\{y \mid A^{\top} y=c^{\top}, y \geq 0\right\}$ are not empty. In addition, the minimum, whenever it is finite, is attained at a vector $y_{V}$ which is a vertex of the polyhedron $\left\{y \mid A^{\top} y=c^{\top}, y \geq 0\right\}$.

We now consider the problem of maximizing the objective linear function

$$
c x:=-x_{s}
$$

over all rational vectors $x, x \in \mathbb{Q}^{n^{\prime}}$, for a suitable $n^{\prime}$, subject to the system of linear inequalities $\mathrm{SLI}\left[Y_{1}\right]$, as an LP-problem $\max \{c x \mid A x \leq b\}$. Note that, in this context, $m^{\prime}=m_{\text {inq }}$ and $n^{\prime}=n_{\text {inq }}$, where $m_{\text {inq }}$ is the number of inequalities in $\operatorname{SLI}\left[Y_{1}\right]$ and $n_{\text {inq }}$ is the number of all variables $x_{j, B}, x_{s}$ in $\operatorname{SLI}\left[Y_{1}\right]$.

It is straightforward to verify that the dual problem

$$
\min \left\{b^{\top} y \mid A^{\top} y=c^{\top}, y \geq 0\right\}
$$

of this LP-problem $\max \{c x \mid A x \leq b\}$ can be equivalently stated as follows

$$
\sum_{i=1}^{m_{\text {inq }}} y_{i} q_{i}^{R} \rightarrow \min \quad \text { subject to } y \geq 0, \sum_{i=1}^{m_{\text {inq }}} y_{i} q_{i}^{L}=-x_{s} .
$$

We emphasize that the last equality should be thought of as a formal linear combination on variables $x_{j, B}, x_{s}$. Note we can write (4.2) in the form

$$
\min \left\{\sum_{i=1}^{m_{\text {inq }}} y_{i} q_{i}^{R} \mid y \geq 0, \sum_{i=1}^{m_{\text {inq }}} y_{i} q_{i}^{L}=-x_{s}\right\} .
$$

In Lemma 3.3, we established the existence of a function

$$
\text { inq : } Y_{2} \mapsto \operatorname{inq}\left(Y_{2}\right) \text {, }
$$


from the set of finite reduced $U_{m}$-graphs $Y_{2}$ with property (B) to a certain set of combinations with repetitions of $\operatorname{SLI}\left[Y_{1}\right]$. Now we will relate these combinations with repetitions of SLI $\left[Y_{1}\right]$ to solutions of the dual LP-problem (4.3).

Consider a combination $Q$ with repetitions of $\operatorname{SLI}\left[Y_{1}\right]$ that has the property that

$$
\sum_{q \in Q} q^{L}=-C(Q) x_{s}
$$

where $C(Q)>0$ is an integer. As above in (4.2)-(4.3), let all of the inequalities in $\operatorname{SLI}\left[Y_{1}\right]$ be indexed and

$$
\mathrm{SLI}\left[Y_{1}\right]=\left\{q_{1}, \ldots, q_{m_{\mathrm{inq}}}\right\} .
$$

Let $\eta_{i}(Q) \geq 0$ denote the number of times that $q_{i}$ occurs in $Q$, and let $\kappa_{i}$ be the coefficient of $x_{s}$ in $q_{i}$. Then it follows from the definitions and (4.4) that

$$
\sum_{q \in Q} q^{L}=\sum_{i=1}^{m_{\text {inq }}} \kappa_{i} \eta_{i}(Q) x_{s}=-C(Q) x_{s} .
$$

Consider the map

$$
\text { sol : } Q \mapsto y_{Q}=\left(y_{Q, 1}, \ldots, y_{Q, m_{\mathrm{inq}}}\right)^{\top},
$$

where $y_{Q, i}:=\frac{\eta_{i}(Q)}{C(Q)}$ for $i=1, \ldots, m_{\text {inq. }}$. It follows from the definitions that $y_{Q}$ is a rational vector, $y_{Q} \geq 0$, and, by (4.5), $y_{Q}$ satisfies the condition that

$$
\sum_{i=1}^{m_{\text {inq }}} y_{Q, i} q_{i}^{L}=-x_{s}
$$

Hence, $y_{Q}$ is a vector in the feasible polyhedron

$$
\left\{y \mid y \geq 0, \sum_{i=1}^{m_{\text {inq }}} y_{i} q_{i}^{L}=-x_{s}\right\}
$$

of the dual LP-problem (4.3).

Note that, in place of (4.6), we could also write

$$
\text { sol : } Q \mapsto C(Q)^{-1} \eta(Q)^{\top},
$$

where $\eta(Q)=\left(\eta_{1}(Q), \ldots, \eta_{m_{\text {inq }}}(Q)\right)$, as $y_{Q}=C(Q)^{-1} \eta(Q)^{\top}$.

Conversely, let $z=\left(z_{1}, \ldots, z_{m_{\text {inq }}}\right)^{\top}$ be a vector of the feasible polyhedron (4.7) of the dual LP-problem (4.3). Let $C>0$ be a common multiple of positive denominators of the rational numbers $z_{1}, \ldots, z_{m_{\text {inq }}}$. Consider a combination with repetitions $Q(z)$ of $\operatorname{SLI}\left[Y_{1}\right]$ such that every $q_{i}$ of $\operatorname{SLI}\left[Y_{1}\right]$ occurs in $Q(z)$ exactly $C z_{i}=n_{i}$ many times. Then it follows from the definitions that

$$
\sum_{q \in Q(z)} q^{L}=\sum_{i=1}^{m_{\text {inq }}} n_{i} q_{i}^{L}=\sum_{i=1}^{m_{\text {inq }}} C z_{i} q_{i}^{L}=C \sum_{i=1}^{m_{\text {inq }}} z_{i} q_{i}^{L}=-C x_{s} .
$$

Now we can see from

$$
\frac{\eta_{i}(Q(z))}{C}=\frac{C z_{i}}{C}=z_{i}
$$

where $i=1, \ldots, m_{\text {inq }}$, that the vector $y_{Q(z)}=\operatorname{sol}(Q(z))$, defined by (4.6) for $Q(z)$, is equal to $z$. 
Lemma 4.1. The map

$$
\text { sol : } Q \mapsto \operatorname{sol}(Q)=y_{Q},
$$

defined by (4.6), is a surjective function from the set of combinations $Q$ with repetitions of $\mathrm{SLI}\left[Y_{1}\right]$ that satisfy the equation $\sum_{q \in Q} q^{L}=-C(Q) x_{s}$, where $C(Q)>0$ is an integer, to the feasible polyhedron (4.7) of the dual LP-problem (4.3). Furthermore, the composition of the maps inq and sol,

$$
\text { sol o inq : } Y_{2} \mapsto \operatorname{sol}\left(\operatorname{inq}\left(Y_{2}\right)\right)=y_{Y_{2}} \text {, }
$$

is a function from the set of graphs with property (B) to the polyhedron (4.7) of (4.3). Under this map, the value of the objective function $\sum_{i=1}^{m_{\text {ina }}} y_{Y_{2}, i} q_{i}^{R}$ of the dual LP-problem (4.3) at $y_{Y_{2}}$ satisfies the equality

$$
\sum_{i=1}^{m_{\text {inq }}} y_{Y_{2}, i} q_{i}^{R}=-\frac{\overline{\mathrm{r}}\left(\operatorname{core}\left(Y_{1} \times Y_{2}\right)\right)}{\overline{\mathrm{r}}\left(Y_{2}\right)} .
$$

In addition, for every $z$ in the polyhedron (4.7), there is a vector $\widetilde{z}$ in (4.7) such that $\widetilde{z}=\operatorname{sol}\left(\operatorname{inq}\left(Y_{2}\right)\right)$ for some graph $Y_{2}$ with property $(B)$ and

$$
\sum_{i=1}^{m_{\text {inq }}} \widetilde{z}_{i} q_{i}^{R} \leq \sum_{i=1}^{m_{\text {inq }}} z_{i} q_{i}^{R}
$$

Proof. As was established above, see computations (4.9)-(4.10), sol is a surjective function.

Consider a finite irreducible $U_{m}$-graph $Y_{2}$ with property (B) and define

$$
Q:=\operatorname{inq}\left(Y_{2}\right), \quad y_{Y_{2}}:=\operatorname{sol}(Q) .
$$

By Lemma 3.3. we have

$$
\sum_{q \in Q} q^{L}=-2 \overline{\mathrm{r}}\left(Y_{2}\right) x_{s} \quad \text { and } \quad \sum_{q \in Q} q^{R}=-2 \overline{\mathrm{r}}\left(\operatorname{core}\left(Y_{1} \times Y_{2}\right)\right) .
$$

It follows from (4.5) and (4.13) that $C(Q)=2 \overline{\mathrm{r}}\left(Y_{2}\right)$. Hence, using the definition (4.6) and equalities (4.13), we obtain

$$
\sum_{i=1}^{m_{\text {inq }}} y_{Y_{2}, i} q_{i}^{R}=\frac{\sum_{q \in Q} q^{R}}{C(Q)}=-\frac{\overline{\mathrm{r}}\left(\operatorname{core}\left(Y_{1} \times Y_{2}\right)\right)}{\overline{\mathrm{r}}\left(Y_{2}\right)},
$$

as required in (4.11).

To prove the additional statement, consider a vector $z$ in the polyhedron (4.7). Since sol is surjective, there is a combination with repetitions $Q$ such that $\operatorname{sol}(Q)=$ $z$. By Lemma 3.3 for this $Q$, there is a graph $Y_{2, Q}$ such that, letting inq $\left(V Y_{2, Q}\right)=\widetilde{Q}$, we have that $|\widetilde{Q}|=|Q|$ and

$$
\begin{aligned}
& \sum_{q \in Q} q^{L}=\sum_{q \in \widetilde{Q}} q^{L}=-2 \overline{\mathrm{r}}\left(Y_{2, Q}\right) x_{s}=-C(Q) x_{s}=-C(\widetilde{Q}) x_{s}, \\
& \sum_{q \in Q} q^{R} \geq \sum_{q \in \widetilde{Q}} q^{R}=-2 \overline{\mathrm{r}}\left(\operatorname{core}\left(Y_{1} \times Y_{2, Q}\right)\right) .
\end{aligned}
$$


Let $\widetilde{z}:=\operatorname{sol}(\widetilde{Q})$. Then, in view of (4.14)- w.15), we obtain

$$
\sum_{i=1}^{m_{\text {inq }}} \widetilde{z}_{i} q_{i}^{R}=\frac{\sum_{q \in \widetilde{Q}} q^{R}}{C(\widetilde{Q})} \leq \frac{\sum_{q \in Q} q^{R}}{C(Q)}=\sum_{i=1}^{m_{\text {inq }}} z_{i} q_{i}^{R},
$$

as required.

We will say that a real nonnegative number $\sigma\left(Y_{1}\right)$ is the WN-coefficient for the graph $Y_{1}$ if

$$
\overline{\mathrm{r}}\left(\operatorname{core}\left(Y_{1} \times Y_{2}\right)\right) \leq \sigma\left(Y_{1}\right) \overline{\mathrm{r}}\left(Y_{1}\right) \overline{\mathrm{r}}\left(Y_{2}\right)
$$

for every finite reduced $U_{m}$-graph $Y_{2}$ and $\sigma\left(Y_{1}\right)$ is minimal with this property.

Lemma 4.2. The $W N$-coefficient $\sigma\left(Y_{1}\right)$ for the graph $Y_{1}$ is equal to

$$
\sup _{Y_{2}}\left\{\frac{\overline{\mathrm{r}}\left(\operatorname{core}\left(Y_{1} \times Y_{2}\right)\right)}{\overline{\mathrm{r}}\left(Y_{1}\right) \overline{\mathrm{r}}\left(Y_{2}\right)}\right\}
$$

over all graphs $Y_{2}$ with property (B).

Proof. Since $\overline{\mathrm{r}}\left(Y_{1}\right)>0$ and $\operatorname{core}\left(Y_{1}\right)=Y_{1}$, we may use $Y_{2}=Y_{1}$ to see that

$$
\sigma\left(Y_{1}\right)=\sup _{Y_{2}} \frac{\overline{\mathrm{r}}\left(\operatorname{core}\left(Y_{1} \times Y_{2}\right)\right)}{\overline{\mathrm{r}}\left(Y_{1}\right) \overline{\mathrm{r}}\left(Y_{2}\right)} \geq \frac{\overline{\mathrm{r}}\left(\operatorname{core}\left(Y_{1} \times Y_{1}\right)\right)}{\overline{\mathrm{r}}\left(Y_{1}\right)^{2}} \geq \frac{1}{\overline{\mathrm{r}}\left(Y_{1}\right)}>0
$$

over all finite reduced $U_{m}$-graph $Y_{2}$ such that $\overline{\mathrm{r}}\left(Y_{2}\right)>0$. We may also assume that $Y_{2}$ has no vertices of degree less than 2, i.e., $\operatorname{core}\left(Y_{2}\right)=Y_{2}$.

Suppose that a graph $Y_{2}$ is such that $\overline{\mathrm{r}}\left(Y_{2}\right)>0$, core $\left(Y_{2}\right)=Y_{2}$ and $Y_{2}$ does not satisfy property $(\mathrm{B})$. This means that the projection

$$
\alpha_{2}: \operatorname{core}\left(Y_{1} \times Y_{2}\right) \rightarrow Y_{2}
$$

is not surjective. We delete those edges and vertices in $Y_{2}$ that have no preimages in core $\left(Y_{1} \times Y_{2}\right)$ under $\alpha_{2}$. As a result, we obtain a subgraph $Y_{2}^{\prime}$ of $Y_{2}$ such that $\operatorname{core}\left(Y_{1} \times Y_{2}^{\prime}\right)=\operatorname{core}\left(Y_{1} \times Y_{2}\right)$ and $0<\overline{\mathrm{r}}\left(Y_{2}^{\prime}\right)<\overline{\mathrm{r}}\left(Y_{2}\right)$. Since

$$
\frac{\overline{\mathrm{r}}\left(\operatorname{core}\left(Y_{1} \times Y_{2}^{\prime}\right)\right)}{\overline{\mathrm{r}}\left(Y_{1}\right) \overline{\mathrm{r}}\left(Y_{2}^{\prime}\right)}>\frac{\overline{\mathrm{r}}\left(\operatorname{core}\left(Y_{1} \times Y_{2}\right)\right)}{\overline{\mathrm{r}}\left(Y_{1}\right) \overline{\mathrm{r}}\left(Y_{2}\right)},
$$

it follows that the graphs $Y_{2}$ that do not satisfy property (B) can be disregarded when taking the supremum (4.16).

Lemma 4.3. Both optima $\max \left\{-x_{s} \mid \operatorname{SLI}\left[Y_{1}\right]\right\}$ and

$$
\min \left\{\sum_{j=1}^{m_{\text {inq }}} y_{j} q_{j}^{R} \mid y \geq 0, \sum_{j=1}^{m_{\text {inq }}} y_{j} q_{j}^{L}=-x_{s}\right\}
$$

are finite and satisfy the following equalities

$$
\begin{aligned}
\max \left\{-x_{s} \mid \operatorname{SLI}\left[Y_{1}\right]\right\} & =\min \left\{\sum_{i=1}^{m_{\text {inq }}} y_{i} q_{i}^{R} \mid y \geq 0, \sum_{i=1}^{m_{\text {inq }}} y_{i} q_{i}^{L}=-x_{s}\right\} \\
& =-\sigma\left(Y_{1}\right) \overline{\mathrm{r}}\left(Y_{1}\right) .
\end{aligned}
$$

Furthermore, the minimum is attained at a vector $\widetilde{y}_{V}$ of the polyhedron (4.7) of the dual LP-problem (4.3) such that there is a graph $Y_{2, Q_{V}}$ with $\operatorname{sol}\left(\operatorname{inq}\left(Y_{2, Q_{V}}\right)\right)=\widetilde{y}_{V}$.

In addition, $\frac{1}{m-2} \leq \sigma\left(Y_{1}\right) \leq 1$. 
Proof. Setting $Y_{2}:=Y_{1}$, we obtain a graph $Y_{2}$ with property (B). Hence, by Lemma 4.1 $y_{Y_{2}}=\operatorname{sol}\left(\operatorname{inq}\left(Y_{2}\right)\right)$ is a solution to the system

$$
y \geq 0, \quad \sum_{j=1}^{m_{\text {inq }}} y_{j} q_{j}^{L}=-x_{s},
$$

so the feasible polyhedron (4.7) of the dual LP-problem (4.3) is not empty.

To establish that the polyhedron $\left\{x \mid \operatorname{SLI}\left[Y_{1}\right]\right\}$ is not empty either, we will show that the vector $\widehat{x}$, whose components are $\widehat{x}_{j, B}:=0$ for all $j=1, \ldots, m$ and for all $B \subseteq E_{a_{j}} Y_{1}$, and $\widehat{x}_{s}:=2 \overline{\mathrm{r}}\left(Y_{1}\right)$, is a solution to $\operatorname{SLI}\left[Y_{1}\right]$. To do this, we need to check that every inequality (3.5)-(3.6) of $\mathrm{SLI}\left[Y_{1}\right]$ is satisfied with these values of variables, that is,

$$
-(k-2) \cdot 2 \overline{\mathrm{r}}\left(Y_{1}\right) \leq-N_{i}\left(A_{1}, \ldots, A_{m}\right)
$$

for every $i$-admissible tuple $\left(A_{1}, \ldots, A_{m}\right)$ in which exactly $k$ sets, among $A_{1}, \ldots, A_{m}$, are nonempty.

It follows from the definition (3.3) of $N_{i}\left(A_{1}, \ldots, A_{m}\right)$ that $N_{i}\left(A_{1}, \ldots, A_{m}\right)=0$ if $k=2$. Hence, if $k=2$ then the inequality (4.18) is true. Since $k \geq 2$, we may assume that $k>2$. Then, according to (3.4), $N_{i}\left(A_{1}, \ldots, A_{m}\right) \leq 2 \overline{\mathrm{r}}\left(Y_{1}\right)$ and the inequality (4.18) is true again.

Hence, both polyhedra

$$
\left\{x \mid \operatorname{SLI}\left[Y_{1}\right]\right\}, \quad\left\{y \mid y \geq 0, \sum_{i=1}^{m_{\text {inq }}} y_{i} q_{i}^{L}=-x_{s}\right\}
$$

are not empty, as required.

According to Theorem A, the maximum and minimum in (4.17) are finite and equal. Referring to Theorem A again, we obtain that the minimum in (4.17) is attained at a vertex $y_{V}$ of the polyhedron (4.7).

It follows from Lemma 4.1 that, for the vertex $y_{V}$, there is a vector $\widetilde{y}_{V}$ in the polyhedron (4.7) such that

$$
\sum_{i=1}^{m_{\text {inq }}} \widetilde{y}_{V, i} q_{i}^{R} \leq \sum_{i=1}^{m_{\text {inq }}} y_{V, i} q_{i}^{R}
$$

and $\widetilde{y}_{V}=\operatorname{sol}\left(\operatorname{inq}\left(Y_{2, Q_{V}}\right)\right)$ for some graph $Y_{2, Q_{V}}$ with property (B). Hence, the minimum in (4.17) is also attained at $\widetilde{y}_{V}$.

By Lemma 4.1, for every graph $Y_{2}$ with property (B), the ratio $-\frac{\overline{\mathrm{r}}\left(\operatorname{core}\left(Y_{1} \times Y_{2}\right)\right)}{\overline{\mathrm{r}}\left(Y_{2}\right)}$ is equal to

$$
\sum_{i=1}^{m_{\text {inq }}} y_{Y_{2}, i} q_{i}^{R}
$$

where $y_{Y_{2}}=\operatorname{sol}\left(\operatorname{inq}\left(Y_{2}\right)\right)$.

On the other hand, it follows from Lemma 4.2 that

$$
\sigma\left(Y_{1}\right) \overline{\mathrm{r}}\left(Y_{1}\right)=\sup _{Y_{2}}\left\{\frac{\overline{\mathrm{r}}\left(\operatorname{core}\left(Y_{1} \times Y_{2}\right)\right)}{\overline{\mathrm{r}}\left(Y_{2}\right)}\right\}=-\inf _{Y_{2}}\left\{\frac{-\overline{\mathrm{r}}\left(\operatorname{core}\left(Y_{1} \times Y_{2}\right)\right)}{\overline{\mathrm{r}}\left(Y_{2}\right)}\right\}
$$

over all graphs $Y_{2}$ with property (B). 
Therefore, putting together these facts, we obtain

$$
\begin{aligned}
-\sigma\left(Y_{1}\right) \overline{\mathrm{r}}\left(Y_{1}\right) & =\inf _{Y_{2}}\left\{\frac{-\overline{\mathrm{r}}\left(\operatorname{core}\left(Y_{1} \times Y_{2}\right)\right)}{\overline{\mathrm{r}}\left(Y_{2}\right)}\right\} \\
& =\inf _{Y_{2}}\left\{\sum_{i=1}^{m_{\text {inq }}} y_{i} q_{i}^{R} \mid y \geq 0, \sum_{i=1}^{m_{\text {inq }}} y_{i} q_{i}^{L}=-x_{s}\right\} \\
& =\min \left\{\sum_{i=1}^{m_{\text {inq }}} y_{i} q_{i}^{R} \mid y \geq 0, \sum_{i=1}^{m_{\text {inq }}} y_{i} q_{i}^{L}=-x_{s}\right\} \\
& =\sum_{i=1}^{m_{\text {inq }}} \widetilde{y}_{V, i} q_{i}^{R}=\max \left\{-x_{s} \mid \operatorname{SLI}\left[Y_{1}\right]\right\},
\end{aligned}
$$

as desired in (4.17). The equalities (4.17) are proven.

It remains to show that $\frac{1}{m-2} \leq \sigma\left(Y_{1}\right) \leq 1$. The inequality $\sigma\left(Y_{1}\right) \leq 1$ follows from the fact that the strengthened Hanna Neumann conjecture is true, see [7], 17, [3, [13.

Let $Y_{2}:=U_{m}$. Then

$$
\overline{\mathrm{r}}\left(Y_{2}\right)=m-2 \quad \text { and } \quad \overline{\mathrm{r}}\left(\operatorname{core}\left(Y_{1} \times Y_{2}\right)\right)=\overline{\mathrm{r}}\left(Y_{1}\right)
$$

because $Y_{1} \times Y_{2}=Y_{1}$. Hence,

$$
\sigma\left(Y_{1}\right) \geq \frac{\overline{\mathrm{r}}\left(\operatorname{core}\left(Y_{1} \times Y_{2}\right)\right)}{\overline{\mathrm{r}}\left(Y_{1}\right) \overline{\mathrm{r}}\left(Y_{2}\right)}=\frac{1}{m-2},
$$

as required.

Lemma 4.4. There exists a finite reduced $U_{m}$-graph $Y_{2, Q_{V}}$ such that $Y_{2, Q_{V}}$ has property (B),

$$
\overline{\mathrm{r}}\left(\operatorname{core}\left(Y_{1} \times Y_{2, Q_{V}}\right)\right)=\sigma\left(Y_{1}\right) \overline{\mathrm{r}}\left(Y_{1}\right) \overline{\mathrm{r}}\left(Y_{2, Q_{V}}\right),
$$

$Y_{2, Q_{V}}$ is connected, and

$$
\left|E Y_{2, Q_{V}}\right|<2^{2^{\left|E Y_{1}\right| / 2+2 \log _{2} m}} .
$$

Proof. According to Lemma 4.3 and to Theorem A, we may assume that the minimum of the dual LP-problem (4.3) is attained at a vertex $y_{V}, y_{V} \geq 0$, of the feasible polyhedron (4.7) of the LP-problem (4.3).

Since $y_{V}$ is a vertex solution of the LP-problem (4.3) and (4.3) is stated in the form $\min \left\{b^{\top} y \mid A^{\top} y=c^{\top}, y \geq 0\right\}$, where $A x \leq b$ is a matrix form of the system (3.7), it follows that the vertex solution $y_{V}$ will satisfy $m_{\text {inq }}$ equalities among

$$
A^{\top} y=c^{\top}, \quad y_{i}=0, \quad i=1, \ldots, m_{\text {inq }},
$$

whose left hand side parts are linearly independent (as formal linear combinations in variables $\left.y_{1}, \ldots, y_{m_{\text {inq }}}\right)$. We will call these $m_{\text {inq }}$ inequalities distinguished.

The foregoing observation implies that there are $r, r \leq m_{\text {inq }}$, distinguished equalities in the system $A^{\top} y=c^{\top}$ such that the submatrix $A_{r}^{\top}$ of $A^{\top}$, consisting of the rows of $A^{\top}$ that correspond to the $r$ distinguished equalities, has the following property. The rank of $A_{r}^{\top}$ is $r$ and deletion of the columns of $A_{r}^{\top}$, that correspond to the variables $y_{i}$ that in turn correspond to the distinguished equalities $y_{i}=0$, produces an $r \times r$ matrix $A_{r \times r}^{\top}$ with $\operatorname{det} A_{r \times r}^{\top} \neq 0$. Reordering the equalities in the 
system $A^{\top} y=c^{\top}$ and variables $y_{i}$ if necessary, we may assume that $A_{r}^{\top}$ consists of the first $r$ rows of $A^{\top}$ and $A_{r \times r}^{\top}$ is an upper left submatrix of $A^{\top}$.

Let

$$
\bar{y}_{V}=\left(y_{V, 1}, \ldots, y_{V, r}\right)
$$

be the truncated version of $y_{V}$ consisting of the first $r$ components. It follows from the definitions that $\bar{y}_{V}$ contains all nonzero components of $y_{V}$ and

$$
A_{r \times r}^{\top} \bar{y}_{V}=\bar{c}^{\top}=\left(c_{1}, \ldots, c_{r}\right)^{\top} .
$$

Since $\sum_{i=1}^{m_{\text {inq }}} y_{V, i} q_{i}^{L}=-x_{s}$, it follows that $c_{j}=0$ if $c_{j}$ corresponds to a variable $x_{\ell, B}$ and $c_{j}=-1$ if $c_{j}$ corresponds to the variable $x_{s}$. Since $y_{V} \neq 0$ following from the definition of the LP-problem (4.3), we conclude that $\bar{c}^{\top} \neq 0$, i.e., one of $c_{j}$ is -1 and all other entries in $\bar{c}^{\top}$ are equal to 0 . Since every entry of $A_{r \times r}$ is 0 or \pm 1 or $-(k-2)$, where $2 \leq k \leq m$, and since every row of $A_{r \times r}$ contains at most $m+1$ nonzero entries, at most one of which is different from \pm 1 , see the definitions (3.5) -(3.6), it follows that the standard Euclidian norm of any row of $A_{r \times r}$ is at most

$$
\left(m+(m-2)^{2}\right)^{1 / 2}<m
$$

as $m \geq 3$. Hence, by the Hadamard's inequality, we obtain

$$
\left|\operatorname{det} A_{r \times r}\right|<m^{r} \text {. }
$$

Invoking the Cramer's rule, we further get that

$$
y_{V, i}=\frac{\operatorname{det} A_{r \times r, i}^{\top}\left(\bar{c}^{\top}\right)}{\operatorname{det} A_{r \times r}},
$$

where $A_{r \times r, i}^{\top}\left(\bar{c}^{\top}\right)$ is the matrix obtained from $A_{r \times r}^{\top}$ by replacing the $i$ th column with $\bar{c}^{\top}, i=1, \ldots, r$. Similarly to (4.19), we have that

$$
\left|\operatorname{det} A_{r \times r, i}\left(\bar{c}^{\top}\right)\right|<m^{r} .
$$

In view of (4.19)-(4.21), we can see that there is a common denominator $C>0$ for the rational numbers $y_{V, 1}, \ldots, y_{V, r}$ that satisfies $C<m^{r}$ and that the nonnegative integers $C y_{V, 1}, \ldots, C y_{V, r}$ are less than $m^{r}$.

It follows from the definition (4.6) of the function sol, see also Lemma 4.1 and (4.9) - (4.10), that if $Q_{V}$ is a combination such that $y_{V}=\operatorname{sol}\left(Q_{V}\right)$ and $\left|Q_{V}\right|$ is minimal with this property, i.e., the entries of $\eta\left(Q_{V}\right)$ are coprime, then

$$
\left|Q_{V}\right|<r m^{r} \text {. }
$$

Recall that the cardinality $|Q|$ of a combination $Q$ is defined so that every $q \in Q$ is counted as many times as it occurs in $Q$.

We now construct a graph $Y_{2, Q_{V}}$ from $Q_{V}$ as described in the proof of Lemma 3.2

It follows from the definitions and Lemmas $4.1,4.3$ that if

$$
\widetilde{y}_{V}:=\operatorname{sol}\left(\operatorname{inq}\left(V Y_{2, Q_{V}}\right)\right)
$$

then the minimum of the dual LP-problem (4.3) is also attained at $\widetilde{y}_{V}$ and this minimum is equal to $-\sigma\left(Y_{1}\right) \overline{\mathrm{r}}\left(Y_{1}\right)$. Hence,

$$
\overline{\mathrm{r}}\left(\operatorname{core}\left(Y_{1} \times Y_{2, Q_{V}}\right)\right)=\sigma\left(Y_{1}\right) \overline{\mathrm{r}}\left(Y_{1}\right) \overline{\mathrm{r}}\left(Y_{2, Q_{V}}\right) .
$$

Since $\left|V Y_{2, Q_{V}}\right|=\left|Q_{V}\right|$, we have from (4.22) that

$$
\left|E Y_{2, Q_{V}}\right| \leq m\left|V Y_{2, Q_{V}}\right|=m\left|Q_{V}\right|<r m^{r+1} \text {. }
$$


Note that $r$ does not exceed the total number $n_{\text {inq }}$ of variables $x_{j, B}, x_{s}$ of SLI $\left[Y_{1}\right]$. It is clear that $\left|E_{a_{j}} Y_{1}\right| \leq\left|E Y_{1}\right| / 2$ for every $j=1, \ldots, m$. Hence, the number of variables $x_{j, B}$ for a fixed $j$ is at most $2^{\left|E Y_{1}\right| / 2}-1$. Therefore,

$$
r \leq n_{\text {inq }} \leq m\left(2^{\left|E Y_{1}\right| / 2}-1\right)+1 \leq m \cdot 2^{\left|E Y_{1}\right| / 2}-2 .
$$

Finally, we obtain from (4.23)-(4.24) that

$$
\begin{aligned}
\left|E Y_{2, Q_{V}}\right|<r m^{r+1} & \leq\left(m \cdot 2^{\left|E Y_{1}\right| / 2}-2\right) m^{m \cdot 2^{\left|E Y_{1}\right| / 2}-1} \\
& <2^{\left|E Y_{1}\right| / 2} m^{m \cdot 2^{\left|E Y_{1}\right| / 2}} \\
& \leq 2^{\left|E Y_{1}\right| / 2+\left(\log _{2} m\right) \cdot m \cdot 2^{\left|E Y_{1}\right| / 2}} \\
& <2^{\left(1+m \cdot \log _{2} m\right) \cdot 2^{\left|E Y_{1}\right| / 2}} \\
& <2^{m^{2} \cdot 2^{\left|E Y_{1}\right| / 2}} \\
& \leq 2^{2^{\left|E Y_{1}\right| / 2+2 \log _{2} m}} .
\end{aligned}
$$

It remains to show that the graph $Y_{2, Q_{V}}$ is connected.

Arguing on the contrary, assume that the graph $Y_{2, Q_{V}}$ is the disjoint union of its two subgraphs $Y_{3}$ and $Y_{4}$. First we assume that

$$
\overline{\mathrm{r}}\left(Y_{3}\right)>0 \quad \text { and } \quad \overline{\mathrm{r}}\left(Y_{4}\right)>0 .
$$

Clearly, $Y_{3}$ and $Y_{4}$ are graphs with property (B). Recall that the vertices of the graph $Y_{2, Q_{V}}$ bijectively correspond to the inequalities of the combination $Q_{V}$, see the proof of Lemma 3.2. In particular, we can consider the combinations $Q_{3}$ and $Q_{4}$, whose inequalities bijectively correspond to the vertices of $Y_{3}$ and $Y_{4}$, resp. It is clear that $Q_{V}$ is the union of combinations $Q_{3}$ and $Q_{4}$ and

$$
\eta\left(Q_{V}\right)=\eta\left(Q_{3}\right)+\eta\left(Q_{4}\right) .
$$

We specify that the union $B_{1} \sqcup B_{2}$ of two combinations $B_{1}, B_{2}$ is the combination whose elements are all elements of both $B_{1}$ and $B_{2}$, in particular, $\left|B_{1} \sqcup B_{2}\right|=$ $\left|B_{1}\right|+\left|B_{2}\right|$.

Furthermore, the graphs $Y_{3}$ and $Y_{4}$ could be constructed from $Q_{3}$ and $Q_{4}$, resp., in the same manner as $Y_{2, Q_{V}}$ was constructed from $Q_{V}$. In particular, the combinations $Q_{3}$ and $Q_{4}$ belong to the domain of the function sol.

Invoking Lemma 4.1 denote $y_{V}(j):=\operatorname{sol}\left(Q_{j}\right), j=3,4$. We also denote

$$
\sum_{q \in Q_{V}} q^{L}=-C\left(Q_{V}\right) x_{s}, \quad \sum_{q \in Q_{j}} q^{L}=-C\left(Q_{j}\right) x_{s},
$$

where $j=3,4$.

Since $Q_{V}=Q_{3} \sqcup Q_{4}$, it follows that $C\left(Q_{V}\right)=C\left(Q_{3}\right)+C\left(Q_{4}\right)$. According to the definition (4.6) of the function sol, we have

$$
y_{V, i}=\frac{\eta_{i}\left(Q_{V}\right)}{C\left(Q_{V}\right)}, \quad y_{V, i}(j)=\frac{\eta_{i}\left(Q_{j}\right)}{C\left(Q_{j}\right)}
$$


for all suitable $i, j$. Hence, in view of (4.27), for every $i=1, \ldots, m_{\text {inq }}$, we obtain

$$
\begin{aligned}
y_{V, i} & =\frac{\eta_{i}\left(Q_{V}\right)}{C\left(Q_{V}\right)}=\frac{\eta_{i}\left(Q_{3}\right)+\eta_{i}\left(Q_{4}\right)}{C\left(Q_{V}\right)} \\
& =\frac{C\left(Q_{3}\right)}{C\left(Q_{V}\right)} \cdot \frac{\eta_{i}\left(Q_{3}\right)}{C\left(Q_{3}\right)}+\frac{C\left(Q_{4}\right)}{C\left(Q_{V}\right)} \cdot \frac{\eta_{i}\left(Q_{4}\right)}{C\left(Q_{4}\right)} \\
& =\lambda_{3} y_{V, i}(3)+\lambda_{4} y_{V, i}(4),
\end{aligned}
$$

where $\lambda_{3}=\frac{C\left(Q_{3}\right)}{C\left(Q_{V}\right)}$ and $\lambda_{4}=\frac{C\left(Q_{4}\right)}{C\left(Q_{V}\right)}$ are positive rational numbers that satisfy $\lambda_{3}+\lambda_{4}=1$.

The equalities (4.29) imply that

$$
y_{V}=\lambda_{3} y_{V}(3)+\lambda_{4} y_{V}(4) .
$$

Since $y_{V}$ is a vertex of the polyhedron (4.7), $y_{V}(3)$ and $y_{V}(4)$ are vectors in (4.7), and $0<\lambda_{3}, \lambda_{4}<1, \lambda_{3}+\lambda_{4}=1$, it follows from (4.30) that

$$
y_{V}(3)=y_{V}(4)=y_{V} \text {. }
$$

Hence, in view of (4.28), the tuples $\eta\left(Q_{V}\right), \eta\left(Q_{3}\right), \eta\left(Q_{4}\right)$ that have integer entries are rational multiples of each other. Referring to (4.27), we conclude that the entries of $\eta\left(Q_{V}\right)$ are not coprime, contrary to the definition of the combination $Q_{V}$. This contradiction completes the case (4.26).

Assume that the graph $Y_{2, Q_{V}}$ is the disjoint union of its two subgraphs $Y_{3}$ and $Y_{4}$ such that

$$
\overline{\mathrm{r}}\left(Y_{3}\right)>0 \quad \text { and } \quad \overline{\mathrm{r}}\left(Y_{4}\right)=0 .
$$

Let $2 Q_{V}$ denote the combination such that $\eta\left(2 Q_{V}\right)=2 \eta\left(Q_{V}\right)$, i.e., to get $2 Q_{V}$ from $Q_{V}$ we double the number of occurrences of each inequality in $Q_{V}$. Using this combination $2 Q_{V}$, we can construct, as in the proof of Lemma 3.2, a graph $Y_{2,2 Q_{V}}$ which consists of two disjoint copies of $Y_{2, Q_{V}}$, denoted $\bar{Y}_{2, Q_{V}}$ and $\widehat{Y}_{2, Q_{V}}$. Since $Y_{2, Q_{V}}=Y_{3} \cup Y_{4}$, we can represent the graph $Y_{2,2 Q_{V}}$ in the form

$$
Y_{2,2 Q_{V}}=Y_{5} \cup Y_{6},
$$

where $Y_{5}:=\bar{Y}_{3} \cup \bar{Y}_{4} \cup \widehat{Y}_{4}$ and $Y_{6}:=\widehat{Y}_{3}$

Clearly, $\overline{\mathrm{r}}\left(Y_{5}\right)>0, \overline{\mathrm{r}}\left(Y_{6}\right)>0$, and both $Y_{5}, Y_{6}$ have property (B). As above, we remark that the vertices of $Y_{2,2 Q_{V}}$ are in bijective correspondence with the inequalities of $2 Q_{V}$. Hence, the combination $2 Q_{V}$ is the union of the combinations $Q_{5}$ and $Q_{6}$ that consist of those inequalities that correspond to the vertices of $Y_{5}$ and $Y_{6}$, resp., and that can be used to construct the graphs $Y_{5}$ and $Y_{6}$ in the same manner as $Y_{2, Q_{V}}$ was constructed from $Q_{V}$.

As above, we can write

$$
\eta\left(2 Q_{V}\right)=\eta\left(Q_{5}\right)+\eta\left(Q_{6}\right) .
$$

Note that the combinations $Q_{5}$ and $Q_{6}$ belong to the domain of the function sol. Using Lemma 4.1 denote $y_{V}(j):=\operatorname{sol}\left(Q_{j}\right), j=5,6$. As above, denote

$$
\sum_{q \in 2 Q_{V}} q^{L}=-C\left(2 Q_{V}\right) x_{s}, \quad \sum_{q \in Q_{j}} q^{L}=-C\left(Q_{j}\right) x_{s},
$$

where $j=5,6$. 
Since $2 Q_{V}=Q_{5} \sqcup Q_{6}$, it follows that $C\left(2 Q_{V}\right)=C\left(Q_{5}\right)+C\left(Q_{6}\right)$. According to the definition (4.6) of the function sol, we have

$$
y_{V, i}=\frac{\eta_{i}\left(Q_{V}\right)}{C\left(Q_{V}\right)}=\frac{\eta_{i}\left(2 Q_{V}\right)}{C\left(2 Q_{V}\right)}, \quad y_{V, i}(j)=\frac{\eta_{i}\left(Q_{j}\right)}{C\left(Q_{j}\right)}
$$

for all suitable $i, j$. Hence, in view of (4.32), for every $i=1, \ldots, m_{\text {inq }}$, we obtain

$$
\begin{aligned}
y_{V, i} & =\frac{\eta_{i}\left(2 Q_{V}\right)}{C\left(2 Q_{V}\right)}=\frac{\eta_{i}\left(Q_{5}\right)+\eta_{i}\left(Q_{6}\right)}{C\left(2 Q_{V}\right)} \\
& =\frac{C\left(Q_{5}\right)}{C\left(2 Q_{V}\right)} \cdot \frac{\eta_{i}\left(Q_{5}\right)}{C\left(Q_{5}\right)}+\frac{C\left(Q_{6}\right)}{C\left(2 Q_{V}\right)} \cdot \frac{\eta_{i}\left(Q_{6}\right)}{C\left(Q_{6}\right)} \\
& =\lambda_{5} y_{V, i}(5)+\lambda_{6} y_{V, i}(6),
\end{aligned}
$$

where $\lambda_{5}=\frac{C\left(Q_{5}\right)}{C\left(2 Q_{V}\right)}$ and $\lambda_{6}=\frac{C\left(Q_{6}\right)}{C\left(2 Q_{V}\right)}$ are positive rational numbers that satisfy $\lambda_{5}+\lambda_{6}=1$.

The equalities (4.34) imply that

$$
y_{V}=\lambda_{5} y_{V}(5)+\lambda_{6} y_{V}(6) .
$$

Since $y_{V}$ is a vertex of the polyhedron (4.7), $y_{V}(5)$ and $y_{V}(6)$ are vectors in (4.7), and $0<\lambda_{5}, \lambda_{6}<1, \lambda_{5}+\lambda_{6}=1$, it follows from (4.35) that

$$
y_{V}(5)=y_{V}(6)=y_{V} .
$$

Hence, in view of (4.33), the tuples $\eta\left(2 Q_{V}\right), \eta\left(Q_{5}\right), \eta\left(Q_{6}\right)$ that have integer entries are rational multiples of each other. Referring to (4.32) and keeping in mind that the entries of $\eta\left(Q_{V}\right)$ are coprime, we conclude that

$$
\eta\left(Q_{V}\right)=\eta\left(Q_{5}\right)=\eta\left(Q_{6}\right),
$$

i.e., $Q_{V}=Q_{5}=Q_{6}$. However, $Y_{6}=\widehat{Y}_{3}$ and $\widehat{Y}_{3}$ is a subgraph of $\widehat{Y}_{2, Q_{V}}$ that consists of several connected components of $\widehat{Y}_{2, Q_{V}}$ and $Y_{3} \neq Y_{2, Q_{V}}$. Hence, $Q_{5} \neq Q_{V}$. This contradiction to (4.36) completes the second case (4.31). Thus the graph $Y_{2, Q_{V}}$ is connected and Lemma 4.4 is proven.

\section{Proofs of Theorems 1.1 and 1.2}

Proof of Theorem 1.1. (a) Suppose that $H_{1}$ is a finitely generated noncyclic subgroup of the free group $F_{U_{m}}=\pi_{1}\left(U_{m}, o_{1}\right)$ of rank $m-1 \geq 2$. Conjugating $H_{1}$ if necessary, we may assume that the reduced $U_{m}$-graph of $H_{1}$, denoted as above by $Y_{1}$, coincides with its core, $\operatorname{core}\left(Y_{1}\right)=Y_{1}$.

As in Section 3, see (3.7), consider a system of linear inequalities SLI $\left[Y_{1}\right]$ with integer coefficients associated with the graph $Y_{1}=Y_{1}\left(H_{1}\right)$ and the LP-problem

$$
\max \left\{-x_{s} \mid \operatorname{SLI}\left[Y_{1}\right]\right\} \text {. }
$$

According to Theorem A and Lemma 4.3, the maximum of the LP-problem (5.1) is equal to $-\sigma\left(Y_{1}\right) \overline{\mathrm{r}}\left(Y_{1}\right)$, as required.

(b) This part follows from the definitions and Lemmas 4.24 .4 applied to the graph $Y_{1}$ of $H_{1}$.

(c) It follows from the definitions of Section 3 that we can algorithmically write down the system SLI $\left[Y_{1}\right]$ and this can be done in exponential time in the size of $Y_{1}$. Note that the size of the graph $Y_{1}$ is polynomial in the size of the input (which is a generating set for $H_{1}$ or the graph $Y_{1}$ itself). It follows from the bound (4.24) and 
from the definitions that the number $n_{\text {inq }}$ of variables of SLI $\left[Y_{1}\right]$ and the number $m_{\text {inq }}$ of inequalities of $\operatorname{SLI}\left[Y_{1}\right]$ satisfy

$$
\begin{gathered}
n_{\text {inq }} \leq m\left(2^{\left|E Y_{1}\right| / 2}-1\right)+1<m 2^{\left|E Y_{1}\right| / 2}, \\
m_{\text {inq }}<2 \cdot 2^{\left|E_{a_{1}} Y_{1}\right|+\cdots+\left|E_{a_{m}} Y_{1}\right|}=2^{\left|E Y_{1}\right| / 2+1} .
\end{gathered}
$$

Furthermore, every number in $\operatorname{SLI}\left[Y_{1}\right]$ is an integer whose absolute value is bounded by $\max \left(m-2,2 \overline{\mathrm{r}}\left(Y_{1}\right)\right)$, see (3.4). Hence, the size of the primal LP-problem $\max \left\{-x_{s} \mid \operatorname{SLI}\left[Y_{1}\right]\right\}$ as well as the size of the dual problem (4.3) are exponential in the size of the input. By Lemma 4.3, the optimal solution to the dual problem (4.3) is equal to

$$
-\sigma\left(Y_{1}\right) \overline{\mathrm{r}}\left(Y_{1}\right)=-\sigma\left(H_{1}\right) \overline{\mathrm{r}}\left(H_{1}\right) .
$$

Since an LP-problem $\max \{c x \mid A x \leq b\}$ can be solved in deterministic polynomial time in the size of the problem, see [21], and since the reduced rank $\overline{\mathrm{r}}\left(Y_{1}\right)=\overline{\mathrm{r}}\left(H_{1}\right)$ can be computed in polynomial time in the size of the input, it follows that the WN-coefficient $\sigma\left(H_{1}\right)$ of $H_{1}$ can be computed in exponential time (in the size of the input).

We recall again that the size of the dual LP-problem (4.3), similarly to the size of the primal LP-problem $\max \left\{-x_{s} \mid \mathrm{SLI}\left[Y_{1}\right]\right\}$, is exponential . Next, a vertex solution $y_{V}$ to the LP-problem (4.3) can be computed in polynomial time in the size of (4.3), see [21. Note that here and below we use the notation of the proof of Lemma 4.4. Hence, a vertex solution $y_{V}$ to (4.3) can be computed in exponential time in the size of $Y_{1}$. Using the function sol, we can compute a combination with repetitions $Q_{V}$, such that $\operatorname{sol}\left(Q_{V}\right)=y_{V}$ and entries of $Q_{V}$ are coprime, in polynomial time in the size of $y_{V}$. The size of the vertex $y_{V}$, as was established in the proof of Lemma 4.4, see (4.19)-(4.21), (4.24), is exponential. Hence, the combination $Q_{V}$ can also be computed in exponential time.

In view of inequalities (4.22) and (4.25), we obtain that

$$
\left|Q_{V}\right|<r m^{r}<2^{2^{\left|E Y_{1}\right| / 2+2 \log _{2} m}} .
$$

This bound, in particular, means that every inequality $q \in \mathrm{SLI}\left[Y_{1}\right]$ occurs in $Q_{V}$ at most $2^{2^{\left|E Y_{1}\right| / 2+2 \log _{2} m}}$ times, hence, the number $n_{Q_{V}}(q)$ of occurrences of $q$ in $Q_{V}$ can be written by using at most $2^{\left|E Y_{1}\right| / 2+2 \log _{2} m}$ bits.

As in the proofs of Lemmas 3.2, 4.4, we construct a graph $Y_{2, Q_{V}}$ whose vertices are in bijective correspondence with inequalities of $Q_{V}$ and whose edges are defined by means of an involution $\iota_{V}$ on the set of terms $\pm x_{j, D}$ of left hand sides $q^{L}$ of the inequalities $q \in Q_{V}$.

Lemma 5.1. The graph $Y_{2, Q_{V}}$ can be constructed in deterministic exponential time in the size of $Y_{1}$.

Proof. We need to explain how to compute the involution $\iota_{V}$ as above in exponential time (in the size of $Y_{1}$ ). To do this, for each variable $x_{j, D}$ of the system $\operatorname{SLI}\left[Y_{1}\right]$, see (3.7), we consider a graph $\Lambda_{j, D}$ whose set of vertices is the subset

$$
R_{V}:=\left\{q \mid q \in Q_{V}\right\}
$$

of SLI $\left[Y_{1}\right]$ formed with the inequalities of $Q_{V}$. If $q_{1}, q_{2} \in R_{V}$ are distinct, $q_{1}^{L}$ contains a term $\pm x_{j, D}$ and $q_{2}^{L}$ contains a term $\mp x_{j, D}$, resp., then we draw an edge $e$ in $\Lambda_{j, D}$ that connects $q_{1}$ and $q_{2}$. In other words, if there is a potential cancellation 
between terms $\pm x_{j, D}$ in the sum $q_{1}^{L}+q_{2}^{L}$ then $\Lambda_{j, D}$ contains an edge that connects $q_{1}$ and $q_{2}$.

It is clear that $\Lambda_{j, D}$ is a bipartite graph so that every edge connects a vertex of type (3.5) and a vertex of type (3.6).

Consider a weight function

$$
\omega_{j, D}: E \Lambda_{j, D} \rightarrow \mathbb{Z}
$$

where $\mathbb{Z}$ is the set of integers, such that $\omega_{j, D}\left(e^{-1}\right)=\omega_{j, D}(e) \geq 0$ and

$$
\sum_{e_{-}=q} \omega_{j, D}(e)=n_{Q_{V}}(q)
$$

where $n_{Q_{V}}(q)$ is the number of occurrences of $q$ in $Q_{V}$.

Our nearest goal is to show that such a weight function $\omega_{j, D}$ can be computed in exponential time for every pair of indices $j, D$. Note that $n_{Q_{V}}(q)=\eta_{i}\left(Q_{V}\right)$ if $q=q_{i}$ in the notation of (4.8).

Let the edge set

$$
E \Lambda_{j, D}=\left\{e_{1}, e_{1}^{-1}, e_{2}, e_{2}^{-1}, \ldots, e_{\left|E \Lambda_{j, D}\right| / 2}, e_{\left|E \Lambda_{j, D}\right| / 2}^{-1}\right\}
$$

of the graph $\Lambda_{j, D}$ be indexed as indicated and let $\left(e_{i}\right)_{-}$be a vertex of type (3.5) for every $i$.

We will define the numbers $\omega_{j, D}\left(e_{i}\right)$ by induction for $i=1,2, \ldots,\left|E \Lambda_{j, D}\right| / 2$ by the following procedure which also assigns intermediate weights $\omega_{j, D}(q)$ to vertices $q \in R_{V}$ of $\Lambda_{j, D}$.

Originally, we set

$$
\omega_{j, D}(q):=n_{Q_{V}}(q)
$$

for every $q \in R_{V}$. For each $i \geq 1$, if the edge $e_{i}$ goes from $q_{1}$ to $q_{2}$ then we set

$$
\omega_{j, D}\left(e_{i}\right):=\min \left(\omega_{j, D}\left(q_{1}\right), \omega_{j, D}\left(q_{2}\right)\right)
$$

and redefine the weights of $q_{1}$ and $q_{2}$ by setting

$$
\begin{aligned}
& \omega_{j, D}^{\prime}\left(q_{1}\right):=\omega_{j, D}\left(q_{1}\right)-\min \left(\omega_{j, D}\left(q_{1}\right), \omega_{j, D}\left(q_{2}\right)\right), \\
& \omega_{j, D}^{\prime}\left(q_{2}\right):=\omega_{j, D}\left(q_{2}\right)-\min \left(\omega_{j, D}\left(q_{1}\right), \omega_{j, D}\left(q_{2}\right)\right),
\end{aligned}
$$

where $\omega_{j, D}^{\prime}\left(q_{1}\right)$ denotes the new weight.

Note that the assignment of a nonnegative weight $\omega_{j, D}\left(e_{i}\right)$ to the edge $e_{i}$, connecting $q_{1}$ and $q_{2}$, can be interpreted as making $\omega_{j, D}\left(e_{i}\right)$ cancellations between terms $\pm x_{j, D}$ of the subsums

$$
\underbrace{q_{1}^{L}+\cdots+q_{1}^{L}}_{n_{Q_{V}}\left(q_{1}\right) \text { times }} \text { and } \underbrace{q_{2}^{L}+\cdots+q_{2}^{L}}_{n_{Q_{V}}\left(q_{2}\right) \text { times }}
$$

of the sum in the left hand side of the equality

$$
\sum_{q \in Q_{V}} q^{L}=-2 \overline{\mathrm{r}}\left(Y_{1}\right) x_{s} .
$$

Analogously, the intermediate weight $\omega_{j, D}\left(q_{1}\right)$ of a vertex $q_{1} \in V \Lambda_{j, D}$ can be interpreted as the number of terms $\pm x_{j, D}$ of the subsum

$$
\underbrace{q_{1}^{L}+\cdots+q_{1}^{L}}_{n_{Q_{V}}\left(q_{1}\right) \text { times }}
$$


which are still uncancelled in the left hand side of (5.4).

Therefore, in view of the equality (5.4), in the end of this process, we will obtain that the weights $\omega_{j, D}(q)$ of all vertices $q \in R_{V}$ are zeros, i.e., cancellations of the terms $\pm x_{j, D}$ are complete, and the weights $\omega_{j, D}\left(e_{i}\right)$ of all edges $e_{i}$ have desired properties.

It is clear that the foregoing inductive procedure makes it possible to compute such a weight function $\omega_{j, D}$ in polynomial time in the size of the graph $\Lambda_{j, D}$ and in the size of numbers $n_{Q_{V}}(q), q \in R_{V}$, written in binary. Hence, we can compute weight functions $\omega_{j, D}$ for all pairs $j, D$ in exponential time in the size of $Y_{1}$.

Now we will define the involution $\iota_{V}$ based on the weight functions $\omega_{j, D}$.

Let elements of the set $R_{V}=\left\{q_{1}, \ldots, q_{\left|R_{V}\right|}\right\}$ be indexed as indicated and let elements of the combination

$$
\begin{aligned}
Q_{V}=[ & {\left[q_{1,1}, q_{1,2}, \ldots, q_{1, n_{Q_{V}}\left(q_{1}\right)},\right.} \\
& q_{2,1}, q_{2,2}, \ldots, q_{2, n_{Q_{V}}\left(q_{2}\right)}, \\
& \ldots, \\
& \left.\left.q_{\left|R_{V}\right|, 1}, q_{\left|R_{V}\right|, 2}, \ldots, q_{\left|R_{V}\right|, n_{Q_{V}}\left(q_{\left|R_{V}\right|}\right)}\right]\right],
\end{aligned}
$$

where $q_{i, \ell}=q_{i} \in R_{V}$ for all possible $i, \ell$, be double indexed as indicated according to the indices introduced on elements of $R_{V}$.

Since the vertices of the graph $Y_{2, Q_{V}}$ are in bijective correspondence with elements of $Q_{V}$, see the proof of Lemma 3.2, we can also write

$$
V Y_{2, Q_{V}}=\left\{u_{i, \ell}|1 \leq i \leq| R_{V} \mid, 1 \leq \ell \leq n_{Q_{V}}\left(q_{i}\right)\right\},
$$

where

$$
u_{i, \ell} \mapsto q_{i, \ell}
$$

under this correspondence.

Let $q_{i} \in R_{V}$ be fixed and let

$$
q_{m_{1}(i)}, \ldots, q_{m_{t_{i}}(i)}
$$

be all the vertices of $\Lambda_{j, D}$, where $m_{1}(i)<\cdots<m_{t_{i}}(i)$, that are connected to $q_{i}$ by edges $f_{1}, \ldots, f_{t_{i}}$, resp., in $\Lambda_{j, D}$ with positive weights $\omega_{j, D}\left(f_{1}\right), \ldots, \omega_{j, D}\left(f_{t_{i}}\right)$, resp. We assume that $q_{i}$ is the terminal vertex of the edges $f_{1}, \ldots, f_{t_{i}}$.

Recall that $q_{i}^{L}$ contains a single term $\pm x_{j, D}$, where the sign is a minus if $q_{i}$ has type (3.5) and the sign is a plus if $q_{i}$ has type (3.6).

According to the weights $\omega_{j, D}\left(f_{1}\right), \ldots, \omega_{j, D}\left(f_{t_{i}}\right)$, we will define $(j, D, i, t)$-blocks of consecutive elements of $Q_{V}$, see (5.5), in the following manner. The $(j, D, i, 1)$ block consists of the first $\omega_{j, D}\left(f_{1}\right)$ elements of the sequence

$$
q_{i, 1}, q_{i, 2}, \ldots, q_{i, n_{Q_{V}}\left(q_{i}\right)} .
$$

The $(j, D, i, 2)$-block consists of the next $\omega_{j, D}\left(f_{2}\right)$ elements of the sequence (5.7) and so on. The $\left(j, D, i, t_{i}\right)$-block consists of the last $\omega_{j, D}\left(f_{t_{i}}\right)$ elements of the sequence (5.7). Since

$$
\sum_{t=1}^{t_{i}} \omega_{j, D}\left(f_{t}\right)=n_{Q_{V}}\left(q_{i}\right)
$$


and $\omega_{j, D}\left(f_{t}\right)>0$ for every $t$, it follows that these $(j, D, i, t)$-blocks, where $t=$ $1, \ldots, t_{i}$ and $j, D, i$ are fixed, will form a partition of the sequence (5.7) into $t_{i}$ subsequences.

We emphasize that every $(j, D, i, t)$-block is associated with a vertex $q_{i} \in V \Lambda_{j, D}=$ $R_{V}$ and with an edge $f_{t}$ of $\Lambda_{j, D}$ so that $f_{t}$ ends in $q_{i}$ and $\omega_{j, D}\left(f_{t}\right)>0$. In particular, for every $(j, D, i, t)$-block, associated with a vertex $q_{i} \in R_{V}$ and with an edge $f_{t}$ of $\Lambda_{j, D}$, we have another $\left(j, D, i^{\prime}, t^{\prime}\right)$-block, associated with a vertex $q_{i^{\prime}} \in R_{V}$ and with an edge $f_{t^{\prime}}^{\prime}$ of $\Lambda_{j, D}$, so that $q_{i^{\prime}} \neq q_{i}$ and $f_{t^{\prime}}^{\prime}=f_{t}^{-1}$. Here $f_{1}^{\prime}, \ldots, f_{t_{i^{\prime}}}^{\prime}$ are the edges of $\Lambda_{j, D}$ defined for $q_{i^{\prime}}$ in the same fashion as the edges $f_{1}, \ldots, f_{t_{i}}$ of $\Lambda_{j, D}$ were defined for $q_{i}$. Note that $i^{\prime \prime}=i$ and $f_{t^{\prime \prime}}^{\prime \prime}=f_{t}$ in this notation.

We define the involution $\iota_{V}$ so that all the terms $\pm x_{j, D}$ of the inequalities of the $(j, D, i, t)$-block are mapped by $\iota_{V}$ to the terms $\mp x_{j, D}$ of the inequalities of the $\left(j, D, i^{\prime}, t^{\prime}\right)$-block in the natural increasing order of elements in the block.

Equivalently, we can say that the vertices

$$
\begin{aligned}
& u_{i, \omega_{j, D}}\left(f_{1}\right)+\cdots+\omega_{j, D}\left(f_{t-1}\right)+1, \\
& u_{i, \omega_{j, D}\left(f_{1}\right)+\cdots+\omega_{j, D}\left(f_{t-1}\right)+2}, \quad \cdots, \\
& u_{i, \omega_{j, D}}\left(f_{1}\right)+\cdots+\omega_{j, D}\left(f_{t}\right),
\end{aligned}
$$

that correspond to $\omega_{j, D}\left(f_{t}\right)$ inequalities of the $(j, D, i, t)$-block, see (5.6), are connected in $Y_{2, Q_{V}}$ to the vertices

$$
\begin{aligned}
& u_{i^{\prime}, \omega_{j, D}}\left(f_{1}^{\prime}\right)+\cdots+\omega_{j, D}\left(f_{t^{\prime}-1}^{\prime}\right)+1, \\
& u_{i^{\prime}, \omega_{j, D}}\left(f_{1}^{\prime}\right)+\cdots+\omega_{j, D}\left(f_{t^{\prime}-1}^{\prime}\right)+2, \quad \ldots, \\
& u_{i^{\prime}, \omega_{j, D}}\left(f_{1}^{\prime}\right)+\cdots+\omega_{j, D}\left(f_{t^{\prime}}^{\prime}\right),
\end{aligned}
$$

resp., that correspond to $\omega_{j, D}\left(f_{t^{\prime}}^{\prime}\right)$ inequalities of the $\left(j, D, i^{\prime}, t^{\prime}\right)$-block, by edges whose labels are $a_{j}$ if $q_{i}$ has type (3.5) or by edges whose labels are $a_{j}^{-1}$ if $q_{i}$ has type (3.6) (here we assume that the edges start at $u_{i, \ell}$ vertices). It is clear that the foregoing construction of the involution $\iota_{V}$ can be done in exponential time. Therefore, the graph $Y_{2, Q_{V}}$ can also be constructed in exponential time, as required. Lemma 5.1 is proved.

Since the graph $Y_{2, Q_{V}}$ can be constructed in exponential time, it follows from Lemma 4.4 that we can output $Y_{2, Q_{V}}$ as the Stallings graph of the desired subgroup $H_{2}^{*}$ of part (b) of Theorem 1.1. The proof of Theorem 1.1 is now complete.

It is worthwhile to mention that our construction of the graph $Y_{2, Q_{V}}$ is somewhat succinct (cf. the definition of succinct representations of graphs in 21]) in the sense that, despite the fact that the size of $Y_{2, Q_{V}}$ could be doubly exponential, we are able to give a description of $Y_{2, Q_{V}}$ in exponential time (in the size of $Y_{1}$ ). In particular, the vertices of $Y_{2, Q_{V}}$ are represented by exponentially long bit strings and the edges of $Y_{2, Q_{V}}$ are drawn in blocks. As a result, we can find out in exponential time whether two given vertices of $Y_{2, Q_{V}}$ are connected by an edge labelled by given letter $a_{j}^{ \pm 1}, a_{j} \in \mathcal{A}$.

Proof of Theorem 1.2. (a) This is immediate from part (c) of Theorem 1.1.

(b) Suppose that a finitely generated subgroup $H$ of the free group $F=\pi_{1}\left(U_{m}, o_{1}\right)$ is not compressed and $K$ is a subgroup of $F$ such that $K$ contains $H$ and $K$ has 
a minimal reduced $\operatorname{rank} \overline{\mathrm{r}}(K)$ such that $\overline{\mathrm{r}}(K)<\overline{\mathrm{r}}(H)$. Let $Y$ and $Z$ be reduced $U_{m}$-graphs of $H$ and $K$, resp.

Since $K$ contains $H$, it follows that there is a locally injective map

$$
\mu: Y \rightarrow Z \text {. }
$$

Suppose that $\mu$ is not surjective. Then there is a subgroup $K^{\prime}$ of $F$, whose graph is $\mu(Y)$, such that $K^{\prime}$ contains $H, K^{\prime}$ is a free factor of $K$ and $\overline{\mathrm{r}}\left(K^{\prime}\right)<\overline{\mathrm{r}}(K)$. This inequality, however, contradicts the minimality of $\overline{\mathrm{r}}(K)$. Hence, the map $\mu$ must be surjective. This means that the graph $Z$ with

$$
\overline{\mathrm{r}}(Z)=\overline{\mathrm{r}}(K)<\overline{\mathrm{r}}(H)=\overline{\mathrm{r}}(Y)
$$

can be obtained from the graph $Y$ by a finite sequence of operations each of which is identification of two edges whose images are equal in $Z$, i.e., the map (5.8) can be factored into a product of maps that go through a finite sequence of graphs (not necessarily reduced) each of which is obtained from the previous one by identification of a pair of edges with the same $\varphi$-label. Note that the edges of such pairs need not have the same initial or terminal vertices.

Therefore, our nondeterministic polynomial time algorithm that verifies whether $H$ is not compressed in $F$ can be run as follows. Starting with the reduced graph $Y$ of $H$, we nondeterministically perform a finite sequence of identification of pairs of edges in $Y$ with the same $\varphi$-label and, if the resulting $U_{m}$-graph $Z$ is reduced and satisfies $\overline{\mathrm{r}}(Z)<\overline{\mathrm{r}}(Y)$, we accept and conclude that $H$ is not compressed. Since each single edge identification decreases the number of edges, it follows that the algorithm runs in nondeterministic linear time in the size of $Y$, as required. Theorem 1.2 is proven.

In conclusion, we mention that it would be of interest to extend our techniques to be able to compute the Hanna Heumann coefficient $\sup _{K}\left\{\frac{\overline{\mathrm{r}}(H \cap K)}{\overline{\mathrm{r}}(H) \overline{\mathrm{r}}(K)}\right\}$ of a finitely generated noncyclic subgroup $H$ of a free group $F$ and to algorithmically decide whether $H$ is inert. However, it is not clear at all how to replace in our arguments the core of the pullback by its single connected component or, alternatively, guarantee, at least in certain situations, that the entire pullback is connected.

Acknowledgements. The author is grateful to the referee for making many helpful remarks and suggestions.

\section{REFERENCES}

[1] S. Arora and B. Barak, Computational complexity - a modern approach, Cambridge Univ. Press, 2009.

[2] W. Dicks, Equivalence of the strengthened Hanna Neumann conjecture and the amalgamated graph conjecture, Invent. Math. 117(1994), 373-389.

[3] W. Dicks, Simplified Mineyev, preprint, http://mat.uab.cat/ dicks/SimplifiedMineyev.pdf

[4] W. Dicks and E. Ventura, The group fixed by a family of injective endomorphisms of a free group, Contemp. Math. 195(1996), 1-81.

[5] W. Dicks and S. V. Ivanov, On the intersection of free subgroups in free products of groups, Math. Proc. Cambridge Phil. Soc. 144(2008), 511-534.

[6] W. Dicks and S. V. Ivanov, On the intersection of free subgroups in free products of groups with no 2-torsion, Illinois J. Math. 54(2010), 223-248. 
[7] J. Friedman, Sheaves on graphs, their homological invariants, and a proof of the Hanna Neumann conjecture: with an appendix by Warren Dicks, Mem. Amer. Math. Soc. 233(2014), no. 1100 . xii+106 pp.

[8] W. Haken, Theorie der Normalflächen, Acta Math. 105(1961), 245-375.

[9] J. Hass, J. C. Lagarias and N. Pippenger, The computational complexity of knot and link problems, J. Assoc. Comput. Mach. 46(1999), 185-211.

[10] G. Hemion, The classification of knots and 3-dimensional spaces, Oxford Univ. Press, 1993.

[11] S. V. Ivanov, On the Kurosh rank of the intersection of subgroups in free products of groups, Adv. Math. 218(2008), 465-484.

[12] S. V. Ivanov, The computational complexity of basic decision problems in 3-dimensional topology, Geom. Dedicata 131(2008), 1-26.

[13] S. V. Ivanov, Intersecting free subgroups in free products of left ordered groups, preprint, arXiv:1607.03010 [math.GR].

[14] S. V. Ivanov, Linear programming and the intersection of free subgroups in free products of groups, preprint, arXiv:1607.03052 [math.GR].

[15] W. H. Jaco and J. L. Tollefson, Algorithms for the complete decomposition of a closed 3-manifold, Illinois J. Math. 39(1995), 358-406.

[16] I. Kapovich and A. G. Myasnikov, Stallings foldings and subgroups of free groups, J. Algebra 248(2002), 608-668.

[17] I. Mineyev, Submultiplicativity and the Hanna Neumann conjecture, Ann. Math. 175(2012), 393-414.

[18] H. Neumann, On the intersection of finitely generated free groups, Publ. Math. 4(1956), 186-189; Addendum, Publ. Math. 5(1957), 128.

[19] W. D. Neumann, On the intersection of finitely generated subgroups of free groups, Lecture Notes in Math. (Groups-Canberra 1989) 1456(1990), 161-170.

[20] C. H. Papadimitriou, Computational complexity, Addison-Wesley Publ., 1994.

[21] A. Schrijver, Theory of linear and integer programming, John Wiley \& Sons, 1986.

[22] J. R. Stallings, Topology of finite graphs, Invent. Math. 71(1983), 551-565.

Department of Mathematics, University of Illinois, Urbana, IL 61801, U.S.A.

E-mail address: ivanov@illinois.edu 